\title{
Zonal Momentum Balance, Potential Vorticity Dynamics, and Mass Fluxes on Near-Surface Isentropes
}

\author{
TAPIO SCHNEIDER \\ California Institute of Technology, Pasadena, California
}

(Manuscript received 2 December 2003, in final form 13 October 2004)

\begin{abstract}
While it has been recognized for some time that isentropic coordinates provide a convenient framework for theories of the global circulation of the atmosphere, the role of boundary effects in the zonal momentum balance and in potential vorticity dynamics on isentropes that intersect the surface has remained unclear. Here, a balance equation is derived that describes the temporal and zonal mean balance of zonal momentum and of potential vorticity on isentropes, including the near-surface isentropes that sometimes intersect the surface. Integrated vertically, the mean zonal momentum or potential vorticity balance leads to a balance condition that relates the mean meridional mass flux along isentropes to eddy fluxes of potential vorticity and surface potential temperature. The isentropic-coordinate balance condition formally resembles balance conditions well known in quasigeostrophic theory, but on near-surface isentropes it generally differs from the quasigeostrophic balance conditions. Not taking the intersection of isentropes with the surface into account, quasigeostrophic theory does not adequately represent the potential vorticity dynamics and mass fluxes on near-surface isentropes - a shortcoming that calls into question the relevance of quasigeostrophic theories for the macroturbulence and global circulation of the atmosphere.
\end{abstract}

\section{Introduction}

Potential vorticity is one of the principal conserved quantities in adiabatic and inviscid atmospheric flows. Considerations of potential vorticity dynamics therefore form a good basis for descriptions of the interaction between a mean flow and rapid, nearly adiabatic and inviscid fluctuations such as occur in baroclinic eddies. Since the potential vorticity flux has only components along isentropes, but not across isentropes (Haynes and McIntyre 1987, 1990), it is convenient to consider the interaction between a mean flow and eddies in isentropic coordinates. For example, on isentropes in the interior of the extratropical atmosphere, the mean meridional mass flux, via the zonal momentum balance, is primarily associated with the meridional eddy flux of potential vorticity (Tung 1986; Yang et al. 1990). For a theory of the mean meridional mass flux along isentropes, or, equivalently, for a theory of the mean meridional entropy flux, one therefore needs a theory of the meridional eddy flux of potential vorticity along isentropes. No conclusive theory has yet been proposed. But a theory of the meridional eddy flux of

Corresponding author address: Tapio Schneider, California Institute of Technology, Mail Code 100-23, 1200 E. California Blvd., Pasadena, CA 91125.

E-mail: tapio@caltech.edu potential vorticity along isentropes-a scalar flux of a quantity that is approximately conserved in baroclinic eddies-seems to be easier to develop than a theory, for instance, of the flux of entropy (or potential temperature), which would necessarily involve vectorvalued eddy fluxes. Since the Eulerian mean mass flux along isentropes approximates the Lagrangian mean mass flux and since it, in concert with radiative processes, determines such basic climatic features as the tropopause height and the thermal stratification of the atmosphere, it has been speculated that a theory of the global circulation of the atmosphere may be based on considerations of potential vorticity dynamics in isentropic coordinates (see, e.g., Hoskins 1991). However, since potential temperature fluctuates at the surface, implying that isentropes sometimes lie above the surface, sometimes "inside" the surface, the role of boundary effects in the zonal momentum balance and in potential vorticity dynamics on near-surface isentropes has remained unclear. Since an understanding of how potential vorticity fluxes along isentropes in the interior atmosphere are related to the zonal momentum balance and to potential vorticity dynamics on nearsurface isentropes has thus far been lacking, it has been impossible to construct a theory of the global circulation of the atmosphere based on considerations of potential vorticity dynamics.

The present paper discusses the temporal and zonal mean balance of zonal momentum and potential vor- 
ticity on isentropes, including the near-surface isentropes that sometimes intersect the surface. A form of the mean zonal momentum balance is derived that holds throughout the flow domain in isentropic coordinates and that is equivalent to the mean potential vorticity balance (section 2). ${ }^{1}$ Integrated vertically, the mean zonal momentum or potential vorticity balance leads to a balance condition that, at each latitude, relates the mean meridional mass flux along isentropes to eddy fluxes of potential vorticity and surface potential temperature (section 3). This balance condition resembles balance conditions well known in quasigeostrophic theory, but on near-surface isentropes it generally differs from the quasigeostrophic balance conditions (section 4).

The developments of this paper presume a hydrostatic ideal-gas atmosphere with stationary flow statistics and with the planet's surface as the only dynamically relevant boundary. The boundary condition at the surface is assumed to be a no-slip condition. The surface can have arbitrary topography, so long as the hydrostatic approximation remains adequate. Appendix A describes an idealized GCM used to illustrate theoretical developments. Appendix B lists the notation and symbols.

\section{Mean balance of zonal momentum in isentropic coordinates}

\section{a. Zonal momentum equation}

The zonal momentum equation in isentropic coordinates can be formulated such that it holds both on isentropes above the surface and on isentropes inside the surface, that is, both on isentropes with potential temperatures $\theta$ greater than or equal to the instantaneous surface potential temperature $\theta_{s}(x, y, t)$ and on isentropes with potential temperatures $\theta$ less than the instantaneous surface potential temperature $\theta_{s}(x, y, t)$. The flow domain in isentropic coordinates can formally be extended to isentropes inside the surface by introducing massless isentropic layers inside the surface (cf. Lorenz 1955). In the definition of the isentropic density $\rho_{\theta}=-\left(g^{-1} \partial_{\theta} p\right) \mathcal{H}\left(\theta-\theta_{s}\right)$ - the mass density in $(x, y, \theta)$ space-one includes the Heaviside step function $\mathcal{H}(\cdot)$ to indicate that the isentropic density vanishes on isentropes inside the surface. With the isentropic density set to zero on isentropes inside the surface, the continuity equation (cf. Andrews et al. 1987, chapter 3.8)

$$
\partial_{t} \rho_{\theta}+\partial_{x}\left(\rho_{\theta} u\right)+\partial_{y}\left(\rho_{\theta} v\right)+\partial_{\theta}\left(\rho_{\theta} Q\right)=0,
$$

with diabatic heating rate $Q=D \theta / D t$, holds on isentropes both above and inside the surface. (Horizontal

\footnotetext{
${ }^{1}$ A form of the mean zonal momentum balance that is essentially equivalent to that presented in section 2 has been independently derived by Koh and Plumb (2004).
}

and time derivatives here are to be understood as derivatives at constant potential temperature.) Similarly, the zonal momentum equation in flux form,

$$
\begin{gathered}
\partial_{t}\left(\rho_{\theta} u\right)-f \rho_{\theta} v+\partial_{x}\left(\rho_{\theta} u^{2}\right)+\partial_{y}\left(\rho_{\theta} u v\right)+\partial_{\theta}\left(\rho_{\theta} u Q\right) \\
=-\rho_{\theta} \partial_{x} M+\rho_{\theta} F^{x},
\end{gathered}
$$

with Montgomery streamfunction $M=c_{p} T+g z$ and with zonal frictional force per unit mass $F^{x}$, holds on isentropes both above and inside the surface (Johnson 1980; Gallimore and Johnson 1981). Combining the continuity equation (1) and the flux-form zonal momentum equation (2), one obtains the form

$$
\rho_{\theta}\left[\partial_{t} u-\left(f+\zeta_{\theta}\right) v\right]=\rho_{\theta}\left(-\partial_{x} B-Q \partial_{\theta} u+F^{x}\right)
$$

of the zonal momentum equation, where $\zeta_{\theta}=\partial_{x} v-\partial_{y} u$ is the relative vorticity component normal to isentropes and

$$
B=\frac{1}{2}\left(u^{2}+v^{2}\right)+M
$$

is the Bernoulli function.

On isentropes above the surface, the isentropic density is greater than zero (provided that the atmosphere is stably stratified, which we assume) and can be dropped from the zonal momentum equation (3). The zonal momentum equation reduces to one of its standard forms (cf. Andrews et al. 1987, appendix 3A). On isentropes inside the surface, the isentropic density vanishes and cannot be dropped from the zonal momentum equation (3). The zonal momentum equation reduces to the trivial statement $0=0$. Although we cannot generally drop the isentropic density from the zonal momentum equation (3), we only need to retain the step function factor to obtain a form of the zonal momentum equation that holds on isentropes both above and inside the surface,

$$
\begin{aligned}
{\left[\partial_{t} u-\left(f+\zeta_{\theta}\right) v\right] \mathcal{H}\left(\theta-\theta_{s}\right) } \\
\quad=\left[-\partial_{x} B-Q \partial_{\theta} u+F^{x}\right] \mathcal{H}\left(\theta-\theta_{s}\right) .
\end{aligned}
$$

We will average this form of the zonal momentum equation to derive a mean balance of zonal momentum on isentropes.

\section{b. Temporal and zonal mean}

When considering mean flows in isentropic coordinates, one must distinguish two regions of the flow domain (cf. Juckes et al. 1994; Held and Schneider 1999). The surface layer at a given latitude is that region of the flow domain in which potential temperatures lie within the range of surface potential temperatures that typically occur at the latitude. The above-lying interior atmosphere is that region of the flow domain in which potential temperatures lie above the range of surface potential temperatures that typically occur at the latitude.

In the interior atmosphere, the step function factor 
$\mathcal{H}\left(\theta-\theta_{s}\right)$ is always equal to one, so the mean acceleration term $\bar{\partial}_{t} u \mathcal{H}\left(\theta-\theta_{s}\right)$ and the mean Bernoulli gradient $\overline{\partial_{x} B \mathcal{H}\left(\theta-\theta_{s}\right)}$ appearing in the isentropic temporal and zonal mean $\overline{(\cdot)}$ of the zonal momentum equation (4) vanish by statistical stationarity and periodicity, respectively. In the surface layer, the mean acceleration term $\overline{\partial_{t} u \mathcal{H}\left(\theta-\theta_{s}\right)}$ is the zonal mean of a normalized sum of differences in zonal velocity between pairs of times at which isentropes "attach to" and "detach from" the surface. Since the velocity vanishes at a no-slip boundary, the mean acceleration term vanishes also in the surface layer. Similarly, the mean Bernoulli gradient $\overline{\partial_{x} B \mathcal{H}\left(\theta-\theta_{s}\right)}$ in the surface layer is the temporal mean of a normalized sum of differences in Bernoulli function between pairs of points at which isentropes "enter" and "leave" the surface. Integrating by parts, using the fact that the distributional derivative of the step function is the Dirac delta function, $\partial_{\xi} \mathcal{H}(\xi)=\delta(\xi)$, and using the no-slip boundary condition to set the Bernoulli function $B=\left(u^{2}+v^{2}\right) / 2+M$ at the surface equal to the Montgomery streamfunction $M$, one can write the mean Bernoulli gradient as

$$
\overline{\partial_{x} B \mathcal{H}\left(\theta-\theta_{s}\right)}=\overline{M \partial_{x} \theta_{s} \delta\left(\theta-\theta_{s}\right)} .
$$

This term generally does not vanish; as we will see, it is essential for the mean zonal momentum balance of the surface layer.

The mean zonal momentum balance takes on a suggestive form if one writes the absolute vorticity $f+\zeta_{\theta}=$ $\rho_{\theta} P$ as the product of isentropic density and potential vorticity $P=\left(f+\zeta_{\theta}\right) / \rho_{\theta}$. Introducing the diabatic component

$$
J_{Q}^{y}=-\rho_{\theta}^{-1} Q \partial_{\theta} u \mathcal{H}\left(\theta-\theta_{s}\right)
$$

and the frictional component

$$
J_{F}^{y}=\rho_{\theta}^{-1} F^{x} \mathcal{H}\left(\theta-\theta_{s}\right)
$$

of the meridional potential vorticity flux (cf. Haynes and McIntyre 1987; Schneider et al. 2003) and averaging the zonal momentum equation (4) leads to

$$
\bar{\rho}_{\theta} \overline{v P}^{*}+\bar{\rho}_{\theta}{\overline{J_{Q}^{y}}}^{*}+\bar{\rho}_{\theta}{\overline{J_{F}^{y}}}^{*}=\overline{M \partial_{x} \theta_{s} \delta\left(\theta-\theta_{s}\right)},
$$

where

$$
\overline{(\cdot)} * \frac{\overline{\left(\rho_{\theta} \cdot\right)}}{\bar{\rho}_{\theta}}
$$

denotes the density-weighted mean associated with the temporal and zonal mean along isentropes. The explicit step function in the advective potential vorticity flux $\overline{v P}^{*}$ was omitted with the understanding that the velocity is taken to vanish on isentropes inside the surface. The left-hand side of the mean zonal momentum balance (6) is generally nonzero both in the interior atmosphere and in the surface layer. The right-hand side is nonzero only in the surface layer.

It is well known that the mean zonal momentum bal- ance (6) on interior isentropes is a statement of mean potential vorticity balance. On interior isentropes, the mean zonal momentum balance can alternatively be derived by averaging the potential vorticity balance (cf. Haynes and McIntyre 1987; Andrews et al. 1987, chapter 3.9). Similarly, the mean zonal momentum balance (6), which holds both on interior and surface layer isentropes, can alternatively be derived by averaging a generalized potential vorticity balance-a potential vorticity balance in which boundary effects are taken into account by generalizing the potential vorticity concept to a sum of the conventional interior potential vorticity and a singular surface potential vorticity (Schneider et al. 2003). ${ }^{2}$ From a generalized potential vorticity perspective, the surface term on the right-hand side of the mean zonal momentum balance (6) arises as the average of a singular surface potential vorticity flux. The mean zonal momentum balance (6), then, can be viewed as a statement of mean potential vorticity balance, not only on interior isentropes, but throughout the flow domain in isentropic coordinates.

\section{c. Vertically integrated mean zonal momentum balance}

How the contribution of the surface term $\overline{M \partial_{x} \theta_{s} \delta\left(\theta-\theta_{s}\right)}$ to the mean zonal momentum or potential vorticity balance (6) is to be interpreted becomes clearer upon vertical integration. Integrating from some nominal lower boundary of the domain at a potential temperature $\theta_{b}$ less than or equal to the lowest potential temperature that occurs at the surface to some isentrope $\theta_{i}$ in the interior atmosphere yields

$$
\int_{\theta_{b}}^{\theta_{i}}\left[\bar{\rho}_{\theta} \overline{v P}^{*}+\bar{\rho}_{\theta}{\overline{J_{Q}^{y}}}^{*}+\bar{\rho}_{\theta}{\overline{J_{F}^{y}}}^{*}\right] d \theta={\overline{M_{s} \partial_{x} \theta_{s}}}^{s} .
$$

Vertically integrated over the surface layer, an isentropic mean $\overline{(\cdot) \delta\left(\theta-\theta_{s}\right)}$ that includes a surface delta function becomes a mean $\overline{(\cdot)_{s}}$ of surface quantities (marked by the subscript $s$ ). Since the mean $\frac{{ }^{2}(\cdot)_{s}}{s}$ of a zonal derivative vanishes, only fluctuations $(\cdot)^{\prime}=(\cdot)-\overline{(\cdot)}^{s}$ about the surface mean contribute to the surface term $\overline{M_{s} \partial_{x} \theta_{s}}=\overline{M_{s}^{\prime} \partial_{x} \theta_{s}^{\prime}}$. Integrating the surface term by parts and introducing a balanced meridional eddy velocity $\tilde{v}_{s}^{\prime}$ at the surface by

$$
\tilde{v}_{s}^{\prime}=f^{-1} \partial_{x}\left(M_{s}^{\prime}-c_{p} \theta_{s}^{\prime}\right)
$$

leads to the vertically integrated mean zonal momentum balance

\footnotetext{
${ }^{2}$ For the derivation of the mean zonal momentum balance (6) from the generalized potential vorticity balance, it is convenient to adopt Gauge I of Schneider et al. (2003). The derivation leads to the same result as the direct derivation from the zonal momentum balance given here.
} 


$$
\int_{\theta_{b}}^{\theta_{i}}\left[\bar{\rho}_{\theta} \overline{v P}^{*}+\bar{\rho}_{\theta}{\overline{J_{Q}^{y}}}^{*}+\bar{\rho}_{\theta}{\overline{J_{F}^{y}}}^{*}\right] d \theta=-f{\overline{\tilde{v}_{s}^{\prime} \theta_{s}^{\prime}}}^{s} .
$$

If fluctuations of surface pressure, temperature, potential temperature, and density about constant reference values $p_{r}, T_{r}, \theta_{r}=T_{r}$, and $\rho_{r}=p_{r} /\left(R T_{r}\right)$ are small, one can approximate surface temperature fluctuations as

$$
\frac{T_{s}^{\prime}}{T_{r}} \approx \kappa \frac{p_{s}^{\prime}}{p_{r}}+\frac{\theta_{s}^{\prime}}{\theta_{r}},
$$

and fluctuations of the Montgomery streamfunction at the surface as

$$
M_{s}^{\prime}=c_{p} T_{s}^{\prime}+g z_{s}^{\prime} \approx \rho_{r}^{-1} p_{s}^{\prime}+g z_{s}^{\prime}+c_{p} \theta_{s}^{\prime} .
$$

The balanced eddy velocity (7) at the surface then is approximately equal to the geostrophic eddy velocity,

$$
\tilde{v}_{s}^{\prime} \approx f^{-1} \partial_{x}\left(\rho_{r}^{-1} p_{s}^{\prime}+g z_{s}^{\prime}\right)=\left.\left(f \rho_{r}\right)^{-1} \partial_{x} p^{\prime}\right|_{z=z_{s}} .
$$

So to the extent that fluctuations of thermodynamic variables about constant reference values are small near the surface, the contribution of the surface term to the vertically integrated mean zonal momentum balance (8) is approximately equal to the geostrophic eddy flux of surface potential temperature, multiplied by the Coriolis parameter.

The balance condition (8) resembles a well-known quasigeostrophic balance condition,

$$
\int_{0}^{\infty}\left(\rho_{0} \overline{v_{g}^{\prime} q^{\prime}}+\rho_{0} \overline{F^{x}}\right) d z=-\left.f_{0} \rho_{0} \frac{\overline{v_{g}^{\prime} \theta^{\prime}}}{\partial_{z} \theta_{0}}\right|_{z=0},
$$

obtained by integrating the quasigeostrophic transformed Eulerian mean of the zonal momentum equation in the vertical and taking into account mass conservation [cf. Andrews and McIntyre (1976); Edmon et al. (1980); Andrews et al. (1987, chapter 3.5)]. Here,

$$
q=f_{0}+\beta_{0} y+\zeta_{g}+\frac{f_{0}}{\rho_{0}} \partial_{z}\left(\rho_{0} \frac{\theta}{\partial_{z} \theta_{0}}\right)
$$

is the quasigeostrophic potential vorticity, $v_{g}$ the geostrophic meridional velocity, and $\zeta_{g}$ the relative vorticity of the geostrophic flow; the subscript 0 marks reference values and profiles; $\overline{(\cdot)} \dot{z}$ denotes the temporal and zonal mean along horizontal planes, and $(\cdot)^{\prime}=(\cdot)-\overline{(\cdot)}^{z}$ denotes fluctuations about this mean. The quasigeostrophic balance condition (9) states that, vertically integrated over an atmospheric column, the flux of quasigeostrophic potential vorticity is balanced by zonal frictional forces and by the geostrophic eddy flux of surface potential temperature, with the momentum flux convergence contained in the quasigeostrophic potential vorticity flux balancing the frictional forces (Green 1970; Held and Hoskins 1985). The diabatic potential vorticity flux $\bar{\rho}_{\theta} \bar{J}_{Q}^{\nu} *=-\overline{Q \partial_{\theta} u \mathcal{H}\left(\theta-\theta_{s}\right)}$ appearing in the isentropic-coordinate balance condition (8) has no counterpart in the quasigeostrophic balance condition (9). It represents the cross-isentropic advection of zonal momentum, whose counterpart-the vertical advection of zonal momentum - is neglected in quasigeostrophic theory.

\section{d. Physical interpretation of surface term}

The surface term $\overline{M \partial_{x} \theta_{s} \delta\left(\theta-\theta_{s}\right)}=\overline{\partial_{x} M \mathcal{H}\left(\theta-\theta_{s}\right)}$ in the mean zonal momentum balance (6) arises from averaging the gradient $\partial_{x} M$ of the Montgomery streamfunction along isentropes that intersect the surface. Since the gradient of the Montgomery streamfunction $\partial_{x} M$ represents minus the zonal pressure force per unit mass in isentropic coordinates, the surface term $\overline{\partial_{x} M \mathcal{H}\left(\theta-\theta_{s}\right)}$ represents a mean zonal pressure drag per unit mass, similar to the pressure drag at mountains appearing in the mean momentum balance in pressure or height coordinates (cf. Peixoto and Oort 1992, chapter 11). Although this mean pressure drag can contain topographic contributions, it does not require topography; it is the mean zonal pressure drag per unit mass that the flow along isentropes experiences at intersections of isentropes with the surface, whether at topographic obstacles or at a flat surface.

Hence, in the vertically integrated zonal momentum balance (8), the term $-f \overline{\tilde{v}_{s}^{\prime} \theta_{s}^{\prime}}$ involving the balanced eddy flux of surface potential temperature is the integral across isentropes of the mean zonal pressure drag per unit mass that the flow along isentropes experiences at the surface. Since this term appears in the vertically integrated zonal momentum balance even if the boundary condition is a no-slip condition, so that the actual eddy flux of surface potential temperature vanishes, the balanced eddy flux of surface potential temperature is not necessarily to be understood as an approximation of the actual eddy flux of surface potential temperature. Even without a small Rossby number approximation, it is the balanced and not the actual eddy flux of surface potential temperature that appears in the vertically integrated zonal momentum balance (8). This suggests that, to the extent that quasigeostrophic potential vorticity dynamics can be interpreted as representing potential vorticity dynamics on isentropes (Charney and Stern 1962), the term involving the geostrophic eddy flux of surface potential temperature in the quasigeostrophic balance condition (9) can likewise be interpreted as a vertical integral of a mean surface pressure drag.

\section{e. Other formulations of mean zonal momentum balance}

Andrews (1983) and Koh and Plumb (2004) have offered similar formulations of the mean zonal momentum balance on isentropes, including isentropes that sometimes intersect the surface.

Andrews' (1983) formulation differs from the present formulation. Andrews introduces fictitious nonzero velocities on isentropes inside the surface and defines the Montgomery streamfunction and its gradient such that 
they are continuous at the intersections of isentropes with the surface. While these conventions allowed him to prove a finite-amplitude Eliassen-Palm theorem, they make it necessary to include in the momentum equation additional forces on isentropes inside the surface. This approach left unclear the relations between the mean zonal momentum balance and the mean potential vorticity balance, and between the vertically integrated potential vorticity flux and the balanced eddy flux of surface potential temperature.

Koh and Plumb (2004) have independently derived a formulation of the mean zonal momentum balance that is essentially equivalent to that presented here, although the equivalence of the formulations may not be obvious because of differences in mathematical techniques, notation, and conventions. Koh and Plumb's mean zonal momentum balance also contains the terms that arise when the velocity at the surface does not vanish-in the present formulation, an acceleration term $\overline{\partial_{t} u \mathcal{H}\left(\theta-\theta_{s}\right)}$ and a kinetic energy term $1 / 2 \overline{\partial_{x}\left(u^{2}+v^{2}\right) \mathcal{H}\left(\theta-\theta_{s}\right)}$ due to the difference between the Bernoulli function and the Montgomery streamfunction at the surface. If the acceleration $\partial_{t} u$ at the surface scales advectively as $U^{2} / L$, with a velocity scale $U$ and a length scale $L$, the acceleration term and the kinetic energy term are of the same order of magnitude and smaller than the pressure drag term $\overline{\partial_{x} M \mathcal{H}\left(\theta-\theta_{s}\right)}$ by a factor of the order of the surface Rossby number $U /(f L)$. Hence, to the extent that the surface Rossby number is small, the acceleration term and the kinetic energy term are negligible, for example, in the mean zonal momentum balance of an atmosphere model that uses a drag-law boundary condition in place of the realistic no-slip boundary condition.

\section{Eddy fluxes and the mean mass flux along isentropes}

\section{a. Derivation of balance condition}

A relationship between the mean mass flux along isentropes and eddy fluxes of potential vorticity and of surface potential temperature can be obtained from the mean zonal momentum or potential vorticity balance (6) if the advective potential vorticity flux $\overline{v P} *=$ $\bar{v}^{*} \bar{P}^{*}+\overline{\hat{v}}^{*}$ is decomposed into a mean advective component $\bar{v}^{*} \bar{P}^{*}$ and an eddy component $\hat{v}^{*}$. [Hats $(\hat{\cdot})=$ $(\cdot)-\overline{(\cdot)} *$ denote fluctuations about the densityweighted mean along isentropes.] Dividing the mean zonal momentum balance (6) by the mean potential vorticity $\bar{P}^{*}$ and rearranging terms yields

$$
\begin{aligned}
\bar{\rho}_{\theta} \bar{v}^{*}= & -\frac{1}{\bar{P}^{*}}\left[\bar{\rho}_{\theta} \overline{\hat{v}}^{*}-\overline{M \partial_{x} \theta_{s} \delta\left(\theta-\theta_{s}\right)}\right. \\
& \left.+\bar{\rho}_{\theta} \bar{J}_{Q}^{y}+\bar{\rho}_{\theta} \bar{J}_{F}^{y}\right] .
\end{aligned}
$$

This form of the mean zonal momentum balance holds where the mean potential vorticity is nonzero. It rep- resents a balance equation that relates the mean mass flux $\bar{\rho}_{\theta} \bar{v}^{*}$ along isentropes to the eddy flux of potential vorticity, to the surface pressure drag, and to the diabatic and frictional components of the potential vorticity flux.

To obtain a balance condition that relates the mean mass flux along isentropes to eddy fluxes of potential vorticity and surface potential temperature, we integrate the balance equation (10) vertically from the lower boundary of the domain at potential temperature $\theta_{b}$ to some isentrope $\theta_{i}$ in the interior atmosphere. The integrated contribution of the surface pressure drag can be simplified by using the expansion

$$
\bar{P}^{*-1}(\theta)=\bar{P}^{*-1}\left(\bar{\theta}_{s}\right)+\partial_{\theta} \bar{P}^{*-1}\left(\bar{\theta}_{s}\right)\left(\theta-\bar{\theta}_{s}\right)+\cdots
$$

for the inverse mean potential vorticity in the surface layer. Keeping only the zeroth-order term and using the relations that led to the vertically integrated zonal momentum balance (8) gives

$$
\begin{aligned}
\int_{\theta_{b}}^{\theta_{i}} \frac{\overline{M \partial_{x} \theta_{s} \delta\left(\theta-\theta_{s}\right)}}{\bar{P}^{*}} d \theta & \approx \frac{1}{\bar{P}^{*}\left(\bar{\theta}_{s}\right)} \int_{\theta_{b}}^{\theta_{i}} \overline{M \partial_{x} \theta_{s} \delta\left(\theta-\theta_{s}\right)} d \theta \\
& =-\frac{f}{\bar{P}^{*}\left(\bar{\theta}_{s}\right)}{\overline{\tilde{v}_{s}^{\prime} \theta_{s}^{\prime}}}^{s}
\end{aligned}
$$

At next order, a term involving the flux $\overline{{\tilde{v}_{s}^{\prime}}^{\prime 2} \theta^{s}}$ of surface potential temperature variance would appear. This term and all other terms involving fluxes of even moments of surface potential temperature fluctuations vanish to leading order for weakly nonlinear quasigeostrophic eddies. For such eddies, the leading-order terms of fluxes of even moments $\overline{\widetilde{v}_{s}^{\prime} \theta_{s}^{\prime 2 n} s}$ have a zonal phase $(\lambda)$ dependence of the form $\sin (\lambda) \cos ^{2 n}(\lambda)(n=$ $1,2, \ldots)$ and so have vanishing phase averages. Hence, although eddies cannot generally be assumed to be quasigeostrophic and weakly nonlinear, the higherorder corrections to the integral (11) are generally smaller than scaling arguments would suggest. Neglecting these higher-order corrections, we obtain

$$
\int_{\theta_{b}}^{\theta_{i}} \bar{\rho}_{\theta} \bar{v}^{*} d \theta \approx-\int_{\theta_{b}}^{\theta_{i}} \frac{\bar{\rho}_{\theta} \overline{\hat{v}}^{*}+\bar{\rho}_{\theta} \bar{J}_{F}^{y}}{\bar{P}^{*}} d \theta-\frac{f}{\bar{P}^{*}\left(\bar{\theta}_{s}\right)}{\overline{\tilde{v}_{s}^{\prime} \theta_{s}^{\prime}}}^{s} .
$$

The diabatic component $\bar{J}_{Q}^{*}$ of the potential vorticity flux, or the cross-isentropic advection of zonal momentum, was neglected because it is smaller than the eddy flux $\hat{v} \hat{P}^{*}$ by a factor of order Rossby number (see, e.g., Tung 1986; Haynes and McIntyre 1987). The balance condition (12) between the integrated mean mass flux along isentropes, on the one hand, and friction and eddy fluxes of potential vorticity and surface potential temperature, on the other hand, holds in the extratropics, where the Rossby number is small and the mean potential vorticity typically is nonzero. The frictional component $-\bar{\rho}_{\theta} \bar{J}_{F}^{y} * / \bar{P}^{*}=-\overline{F^{x} \mathcal{H}\left(\theta-\theta_{s}\right)} / \bar{P}$ of the mass 
flux is an Ekman mass flux. If the top of the atmosphere is taken as the upper limit $\theta_{i}$ of the integrals, the lefthand side of the balance condition (12) is zero by mass conservation, so the balance condition (12) becomes a balance condition between friction and eddy fluxes of potential vorticity and surface potential temperature.

Using more restrictive assumptions, Held and Schneider (1999) argued that, in the extratropics, the mean mass flux along isentropes integrated over the surface layer contains a component proportional to the geostrophic eddy flux of surface potential temperature. The balance condition (12) shows in more detail that not only the balanced eddy flux $\overline{\tilde{v}_{s}^{\prime} \theta_{s}^{\prime}} s$ of surface potential temperature and the frictional component $\bar{J}_{F}^{y} *$ of the potential vorticity flux, but also the eddy flux $\hat{v} \hat{P}^{*}$ of potential vorticity contribute to the mean mass flux integrated over the surface layer.

\section{b. Conventions for potential vorticity in surface layer}

The contribution of isentropes inside the surface to the mean potential vorticity $\bar{P}^{*}=\overline{\left[\rho_{\theta}\left(f+\zeta_{\theta}\right) / \rho_{\theta}\right]} / \bar{\rho}_{\theta}$ involves the indefinite expression $\left(f+\zeta_{\theta}\right) \rho_{\theta} / \rho_{\theta}$. The total potential vorticity flux $\overline{v P}$ is independent of the conventions one adopts to assign values to this indefinite expression. But the mean potential vorticity $\bar{P}^{*}$ in the surface layer, and thus the eddy flux of potential vorticity $\hat{v} \hat{P}^{*}=\overline{v P}^{*}-\bar{v}^{*} \bar{P}^{*}$, depend on conventions. The magnitudes of the Ekman mass flux and of the mass fluxes associated with the eddy fluxes of potential vorticity and surface potential temperature in the balance condition (12) likewise depend on conventions.

\section{1) Convention I}

Under convention I, the absolute vorticity on isentropes inside the surface equals the Coriolis parameter, $\rho_{\theta} P=f+\zeta_{\theta}=f$. One construal of this convention is that, on isentropes inside the surface, the Coriolis parameter $f$ takes on its usual value, the relative vorticity $\zeta_{\theta}$ vanishes, and the indefinite expression $\rho_{\theta} / \rho_{\theta}$ equals one.

Convention I is convenient for closures of the eddy flux of potential vorticity in the surface layer because, under convention I, the mean potential vorticity gradient in the surface layer is usually well defined and varies on large scales. Under convention I, the mean potential vorticity throughout the atmosphere, including the surface layer, can be written as $\bar{P}^{*}=\left(f+\bar{\zeta}_{\theta}\right) / \bar{\rho}_{\theta}$, and, if the relative vorticity gradient can be neglected, the mean potential vorticity gradient can be approximated by

$$
\partial_{y} \bar{P}^{*} \approx \frac{\beta}{\bar{\rho}_{\theta}}-f \frac{\partial_{y} \bar{\rho}_{\theta}}{\bar{\rho}_{\theta}^{2}} .
$$

The first term on the right-hand side is positive both in the interior atmosphere and in the surface layer. The second term typically changes sign near the top of the

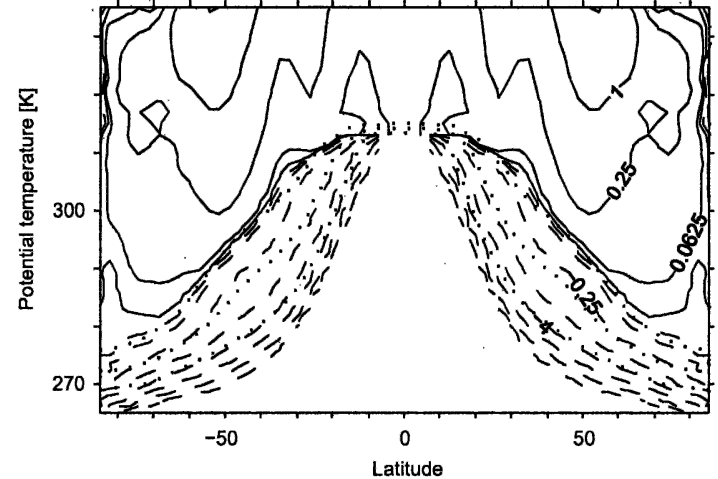

FIG. 1. Mean potential vorticity gradient $\partial_{y} \bar{P}^{*}\left(10^{-6} \mathrm{PVU} \mathrm{m}^{-1}\right.$, with $1 \mathrm{PVU}=10^{-6} \mathrm{~m}^{2} \mathrm{~s}^{-1} \mathrm{~K} \mathrm{~kg}^{-1}$ ) under convention I. The contouring is logarithmic, with contour levels at $\pm\left(4^{-3}, 4^{-2}, \ldots\right.$, $\left.4^{4}\right) \times 10^{-6} \mathrm{PVU} \mathrm{m}^{-1}$. Here and in subsequent figures, the dotted lines represent the $10 \%, 50 \%$, and $90 \%$ isolines of the cumulative distribution of surface potential temperatures.

surface layer: it is usually positive in the interior troposphere, where the mean isentropic density decreases poleward along isentropes; it is usually negative in the surface layer, where the mean isentropic density increases poleward along isentropes. The mean isentropic density increases poleward along surface-layer isentropes primarily because a pole-to-equator surface potential temperature gradient implies that the relative frequency with which an isentrope lies above the surface increases poleward along the isentrope. (If variations of the density and static stability near the surface are ignored, the mean isentropic density $\bar{\rho}_{\theta} \propto \overline{\mathcal{H}\left(\theta-\theta_{s}\right)}$ in the surface layer is proportional to the cumulative distribution of surface potential temperatures $\Pi(y, \theta)=$ $\overline{\mathcal{H}\left(\theta-\theta_{s}\right)}$, or to the relative frequency with which an isentrope lies above the surface.) If the variance and higher moments of surface potential temperature fluctuations vary on much larger meridional scales than the mean surface potential temperature, the isentropic gradient $\partial_{y} \Pi$ of the cumulative distribution of surface potential temperatures is directly related to the mean surface potential temperature gradient, which is usually well defined and varies on large scales. To the extent that the term involving $\beta$ is small, this implies that the gradient (13) of the mean potential vorticity in the surface layer is usually negative and likewise varies on large scales.

Figure 1 shows the mean potential vorticity gradient under convention $I$ in a simulation with an idealized GCM (described in appendix A). Included in Fig. 1 are the $10 \%, 50 \%$, and $90 \%$ isolines of the cumulative distribution of surface potential temperatures. The $50 \%$ isoline, the median, approximates the mean surface potential temperature, and the $10 \%$ and $90 \%$ isolines can be taken as demarcating the surface layer. The figure shows that the mean potential vorticity gradient is indeed positive in the interior troposphere and negative in the surface layer and that it varies on large scales. 
If the mean potential vorticity gradient varies on meridional scales that are large in comparison with eddy length scales-an acceptable albeit not very accurate assumption in the surface layer-terms involving third and higher derivatives in an expansion of the eddy flux of potential vorticity $\hat{\hat{v}}^{*}$ in terms of meridional derivatives of the mean potential vorticity can be neglected (terms involving even derivatives vanish by symmetry). The eddy flux ${ }_{*}$ can then be modeled using a diffusive closure $\hat{v} \hat{P}^{*} \approx-D \partial_{y} \bar{P}^{*}$ with an eddy diffusivity $D$ (Corrsin 1974). In the simulation shown here and in other simulations with the idealized GCM, the empirical eddy diffusivities $-\overline{\hat{v}}^{*} / \partial_{y} \bar{P}^{*}$ in the surface layer indeed vary on large scales under convention I and are positive, except near the top of the surface layer, where the mean potential vorticity gradient changes sign and eddy diffusivities are not defined.

The approximation (11) of the integrated surface pressure drag contribution to the mass flux along isentropes appears to be very accurate under convention I. In the simulation with the idealized GCM, it entails errors of $5 \%-10 \%$ in midlatitudes. In other simulations with the idealized GCM, spanning a wide range of climates, the approximation (11) consistently entails midlatitude errors of about $10 \%$ and often less. ${ }^{3}$

Using convention I and the approximation of small Rossby number,

$$
\bar{P}^{*}\left(\bar{\theta}_{s}\right)=\left.\frac{\overline{f+\zeta_{\theta}}}{\bar{\rho}_{\theta}}\right|_{\bar{\theta}_{s}} \approx \frac{f}{\bar{\rho}_{\theta}^{0}},
$$

where $\bar{\rho}_{\theta}^{0}=\bar{\rho}_{\theta}\left(\bar{\theta}_{s}\right)$ denotes the isentropic density at the mean surface potential temperature $\bar{\theta}_{s}(y)$, one can write the balance condition (12) as

$$
\int_{\theta_{b}}^{\theta_{i}} \bar{\rho}_{\theta} \bar{v}^{*} d \theta \approx-\int_{\theta_{b}}^{\theta_{i}} \frac{\bar{\rho}_{\theta} \overline{\hat{v}}^{*}+\bar{\rho}_{\theta}{\overline{J_{F}^{y}}}^{*}}{\bar{P}^{*}} d \theta-\bar{\rho}_{\theta}^{0}{\overline{\tilde{v}_{s}^{\prime} \theta_{s}^{\prime}}}^{s} .
$$

Schneider (2004) takes this balance condition as the point of departure for the derivation of a constraint on the tropopause height and the thermal stratification of the troposphere.

\footnotetext{
${ }^{3}$ To the extent that eddies are weakly nonlinear and quasigeostrophic and that the distribution of surface potential temperature fluctuations is symmetric about the mean surface potential temperature, heuristic arguments may account for the accuracy of the approximation (11) under convention I. For weakly nonlinear quasigeostrophic eddies, the fluxes of even moments of surface potential temperature fluctuations vanish to leading order, and for a symmetric distribution of surface potential temperature fluctuations, the second derivative $\partial_{\theta \theta} \Pi$ of the cumulative distribution function vanishes at the mean surface potential temperature. So if the Rossby number is small $\left(\bar{P}^{*} \approx f / \bar{\rho}_{\theta}\right)$ and if the mean isentropic density in the surface layer can be approximated by $\bar{\rho}_{\theta} \propto \Pi$, the first correction to the zeroth-order term in the integral (11) may only be the fourth-order term involving the flux of the fifth moment of surface potential temperature fluctuations.
}

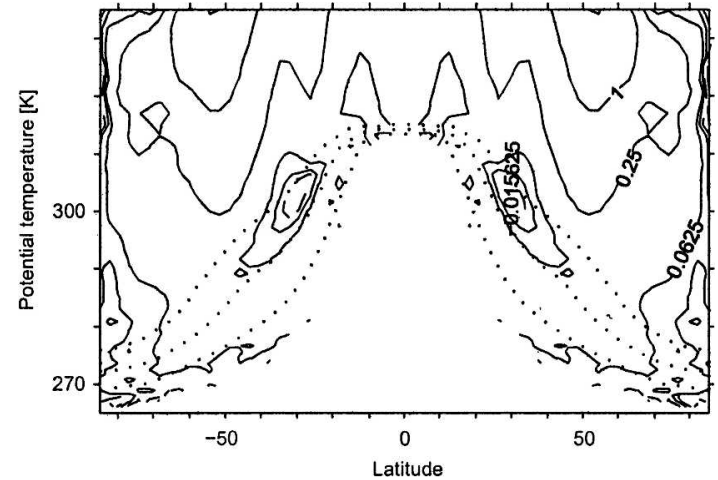

FIG. 2. Mean potential vorticity gradient $\partial_{y} \bar{P}^{*}\left(10^{-6} \mathrm{PVU} \mathrm{m}{ }^{-1}\right)$ under convention II. The contouring is logarithmic, with contour levels at $\pm\left(4^{-3}, 4^{-2}, \ldots, 4^{4}\right) \times 10^{-6} \mathrm{PVU} \mathrm{m}^{-1}$.

\section{2) Convention II}

Under convention II, the absolute vorticity on isentropes inside the surface equals zero, $\rho_{\theta} P=f+\zeta_{\theta}=0$. One construal of this convention is that the indefinite expression $\rho_{\theta} / \rho_{\theta}$ vanishes on isentropes inside the surface. Schneider et al. (2003) and Koh and Plumb (2004) adopted this convention in their discussions of the potential vorticity budget on isentropes.

Convention II is less convenient for closures of the eddy flux of potential vorticity in the surface layer than convention I because, under convention II, the mean potential vorticity gradient in the surface layer does not necessarily have a definite sign and can vary on relatively small scales. Under convention II, the mean potential vorticity can be written as $\bar{P}^{*}=\left(f \Pi+\bar{\zeta}_{\theta}\right) / \bar{\rho}_{\theta}$, with the understanding that the relative vorticity vanishes on isentropes inside the surface. If the relative vorticity gradient can be neglected, the mean potential vorticity gradient

$$
\partial_{y} \bar{P}^{*} \approx \frac{\beta \Pi}{\bar{\rho}_{\theta}}-f \Pi \frac{\partial_{y} \bar{\rho}_{\theta}}{\bar{\rho}_{\theta}^{2}}+\frac{f}{\bar{\rho}_{\theta}} \partial_{y} \Pi
$$

involves a third term proportional to the gradient of the cumulative distribution of surface potential temperatures. If the mean isentropic density in the surface layer is proportional to the cumulative distribution function, $\bar{\rho}_{\theta} \propto \Pi$, this third term cancels the second term on the right-hand side. But to the extent that the cancellation is incomplete and that the term involving $\beta$ is small, the mean potential vorticity gradient under convention II does not necessarily have a definite sign and can vary on relatively small scales.

Figure 2 shows the mean potential vorticity gradient under convention II in the simulation with the idealized GCM. The mean potential vorticity gradient in the surface layer varies on smaller scales under convention II than under convention I. Correspondingly, in the simulation shown here and in other simulations with the 
idealized GCM, empirical eddy diffusivities $-\overline{\hat{v}}^{*} / \partial_{y} \bar{P}^{*}$ for potential vorticity in the surface layer vary on smaller scales under convention II than under convention I and even are, in some instances, negative under convention II.

If the mean surface potential temperature is approximately equal to the median surface potential temperature, the mean potential vorticity at the mean surface potential temperature is about a factor of 2 smaller under convention II than under convention I: $\bar{P}^{*}\left(\bar{\theta}_{s}\right) \approx$ $f \Pi\left(\bar{\theta}_{s}\right) / \bar{\rho}_{\theta}^{0} \approx 0.5 f / \bar{\rho}_{\theta}^{0}$. Therefore, the mass flux associated with the eddy flux of surface potential temperature on the right-hand side of the balance condition (12) is about a factor of 2 greater under convention II than under convention I. The other terms on the right-hand side of the balance condition (12) have correspondingly changed magnitudes under convention II, and the mass flux associated with the eddy flux of potential vorticity in the surface layer can have a different sign under convention II than under convention I.

The approximation (11) of the integrated surface pressure drag contribution to the mass flux along isentropes also appears to be accurate under convention II, albeit less so than under convention I. In the simulation with the idealized GCM, the approximation (11) entails errors of $10 \%-30 \%$ in midlatitudes. Since the mass flux associated with the integrated surface pressure drag is about a factor of 2 greater under convention II, the fact that the relative error of the approximation (11) is about a factor of 2-3 greater translates into an absolute error that is about a factor of 4-6 greater than under convention I. In other simulations with the idealized GCM, the absolute error of the approximation (11) is consistently larger under convention II than under convention $\mathrm{I}$.

Conventions I and II are two possible conventions for the mean potential vorticity in the surface layer. Other conventions, assigning other values to the absolute vorticity $\rho_{\theta} P$ on isentropes inside the surface, are possible in principle. The analyses here will be focused on convention I, since it is convenient for eddy flux closures and since it will allow us to draw analogies between the isentropic-coordinate surface layer and the lower layer of quasigeostrophic two-layer models (section $4 \mathrm{~b}$ ). However, since the quasigeostrophic limit of the balance condition (12) is obtained under convention II (section 4a), some results under convention II that are pertinent to the quasigeostrophic limit will also be discussed.

\section{c. Geostrophic limit of mean zonal momentum balance}

To interpret the balance condition (15) in terms of the mean zonal momentum balance on isentropes and to make connections with quasigeostrophic theory, it is helpful to consider the geostrophic limit. Introducing the geostrophic meridional velocity in isentropic coordinates by $v_{\mathrm{g}}=f^{-1} \partial_{x} M \mathcal{H}$, with the shorthand $\mathcal{H}=$
$\mathcal{H}\left(\theta-\theta_{s}\right)$ for the step function, we can write the density-weighted mean of the geostrophic balance equation as

$$
-f \bar{\rho}_{\theta} \bar{v}_{g}^{*}=-\overline{\rho_{\theta} \partial_{x} M} .
$$

In this form, geostrophic balance states that the Coriolis force on the geostrophic mass flux along an isentrope balances the zonal pressure gradient force, which, in turn, is equal to the form drag exerted on the isentrope (Andrews 1983; Held and Schneider 1999; Koh and Plumb 2004). The form drag includes the surface pressure drag that the flow along isentropes experiences at intersections of isentropes with the surface. Decomposing the geostrophic mass flux and the zonal pressure gradient force into mean and eddy components,

$$
f \bar{\rho}_{\theta} \bar{v}_{g}=\bar{\rho}_{\theta} \overline{\partial_{x} M \mathcal{H}} \text { and } f \overline{\rho_{\theta}^{\prime} v_{g}^{\prime}}=\overline{\rho_{\theta}^{\prime}\left(\partial_{x} M \mathcal{H}\right)^{\prime}} \text {, }
$$

with primes here denoting fluctuations $(\cdot)^{\prime}=(\cdot)-\overline{(\cdot)}$ about the isentropic mean, one can alternatively write the geostrophic balance equation as

$$
\bar{\rho}_{\theta} \bar{v}_{g}^{*}=\overline{\rho_{\theta}^{\prime} v_{g}^{\prime}}+\bar{\rho}_{\theta} \bar{v}_{g}=f^{-1} \overline{\rho_{\theta} \partial_{x} M} .
$$

In the surface layer, unlike in the interior atmosphere, the mean $\bar{v}_{g}$ of the geostrophic velocity is generally nonzero.

In the geostrophic limit, the vertical integrals of the eddy component $\overline{\rho_{\theta}^{\prime} v_{g}^{\prime}}$ and of the mean component $\bar{\rho}_{\theta} \bar{v}_{g}$ of the geostrophic mass flux can be identified with the two eddy flux terms on the right-hand side of the balance condition (15). To consider this limit, we neglect relative vorticity contributions to the potential vorticity, writing $P_{g}=f / \rho_{\theta}$, and we replace velocities by geostrophic velocities.

(i) In the geostrophic limit, potential vorticity fluctuations about the density-weighted isentropic mean can be written as $\hat{P}_{g}=f\left(\rho_{\theta}^{-1}-\bar{\rho}_{\theta}^{-1}\right)$. Upon multiplication by the isentropic density, it follows that $\rho_{\theta} \hat{P}_{g}=-\left(f / \bar{\rho}_{\theta}\right) \rho_{\theta}^{\prime}$. Since, for isentropic means of quadratic terms, the relation $\overline{(\cdot)(\cdot)^{\prime}}=\overline{(\cdot)^{\prime}(\cdot)^{\prime}}$ holds (cf. Tung 1986), the mass flux associated with the eddy flux of potential vorticity is, in the geostrophic limit, equal to the eddy mass flux,

$$
-\frac{\bar{\rho}_{\theta} \overline{\hat{v}}_{g}^{*}}{\bar{P}_{g}^{*}}=\overline{\rho_{\theta}^{\prime} v^{\prime}},
$$

or, upon substitution of geostrophic velocities,

$$
-\frac{\bar{\rho}_{\theta}{\overline{\hat{v}_{g}}}_{\hat{P}_{g}^{*}}^{*}}{\bar{P}_{g}^{*}}=\overline{\rho_{\theta}^{\prime} v_{g}^{\prime}} .
$$

Thus, the mass flux associated with the eddy flux of potential vorticity that appears in the balance condition (15) is, in the geostrophic limit, equal to the geostrophic eddy mass flux. From the perspective of the zonal momentum balance, this implies that, in the geostrophic limit, the Coriolis force on the 
mass flux associated with the eddy flux of potential vorticity balances an eddy form drag.

The relation (16b) between geostrophic eddy fluxes of potential vorticity and mass has a counterpart in quasigeostrophic theory. If the contribution of the relative vorticity to the quasigeostrophic potential vorticity is neglected, the mass flux associated with the quasigeostrophic potential vorticity flux is likewise equal to the geostrophic eddy mass flux (cf. Rhines and Holland 1979). Unlike in quasigeostrophic theory, however, in isentropic coordinates it is only necessary to assume that the contribution of the relative vorticity to the potential vorticity is small; it is not necessary to assume that fluctuations of the isentropic density are small.

(ii) In the geostrophic limit, the term $\overline{\partial_{x} M \mathcal{H}} / \bar{P}^{*}=$ $\overline{M \partial_{x} \theta_{s} \delta\left(\theta-\theta_{s}\right)} / \bar{P}^{*}$ in the zonal momentum balance (10) becomes $\bar{\rho}_{\theta} \overline{\partial_{x} M \mathcal{H}} / f=\bar{\rho}_{\theta} \bar{v}_{g}$. The approximate vertical integral (11) of this term is what gave rise to the mass flux associated with the balanced eddy flux of surface potential temperature,

$$
\int_{\theta_{b}}^{\theta_{i}} \bar{\rho}_{\theta} \bar{v}_{g} d \theta \approx-\bar{\rho}_{\theta}^{0} \overline{\tilde{v}_{s}^{\prime} \theta_{s}^{s}} .
$$

Thus, upon vertical integration over the surface layer, the geostrophic mean mass flux is approximately equal to the mass flux associated with the balanced eddy flux of surface potential temperature that appears in the balance condition (15). From the perspective of the zonal momentum balance, this implies that, in the geostrophic limit and upon vertical integration, the Coriolis force on the mass flux associated with the balanced eddy flux of surface potential temperature approximately balances a mean form drag, or a mean surface pressure drag due to the intersection of isentropes with the surface.

These relations between geostrophic eddy and mean mass fluxes and eddy fluxes of potential vorticity and surface potential temperature hold under convention I for the potential vorticity.

Under convention II, one can derive similar relations between geostrophic eddy and mean mass fluxes and eddy fluxes of potential vorticity and surface potential temperature if one introduces a mean

$$
\overline{(\cdot)} \diamond=\frac{\overline{(\cdot \mathcal{H})}}{\Pi}
$$

that is normalized by the relative frequency with which an isentrope lies above the surface and if one defines eddy fields $(\cdot)=(\cdot)-\overline{(\cdot)}$ as fluctuations about this mean. Reasoning analogous to the above then leads to the relation

$$
-\frac{\bar{\rho}_{\theta}{\overline{\hat{v}_{g}}}_{\hat{P}_{g}}^{*}}{\bar{P}_{g}^{*}}=\Pi{\overline{\check{\rho}_{\theta}}}_{\check{v}_{g}} \diamond
$$

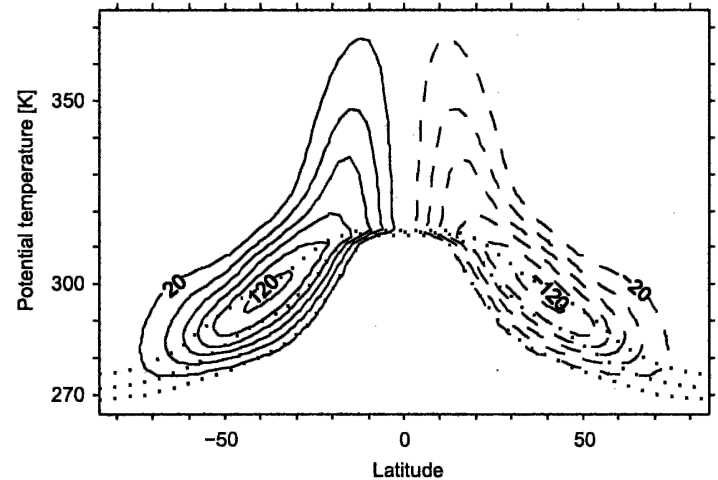

FIG. 3. Mass flux streamfunction $\Psi\left(10^{9} \mathrm{~kg} \mathrm{~s}^{-1}\right)$ (solid lines: counterclockwise rotation; dashed lines: clockwise rotation).

between the mass flux associated with the eddy flux of potential vorticity and a geostrophic eddy mass flux. The mean geostrophic mass flux is related to the balanced eddy flux of surface potential temperature by

$$
\int_{\theta_{b}}^{\theta_{i}} \bar{\rho}_{\theta}^{\diamond} \bar{v}_{g} d \theta \approx-\bar{\rho}_{\theta}^{\diamond}\left(\bar{\theta}_{s}\right) \overline{\tilde{v}_{s}^{\prime} \theta_{s}^{\prime}},
$$

where $\bar{\rho}_{\theta}^{\diamond}\left(\bar{\theta}_{s}\right)=\bar{\rho}_{\theta}^{0} / \Pi\left(\bar{\theta}_{s}\right) \approx 2 \bar{\rho}_{\theta}^{0}$ is the mean isentropic density at the mean surface potential temperature, normalized by the value of the cumulative distribution function $\Pi\left(\bar{\theta}_{s}\right) \approx 0.5$. In the geostrophic limit under convention II, the vertical integrals of the eddy component $\Pi \grave{\rho}_{\theta} \breve{v}_{g} \diamond$ and of the mean component $\Pi \bar{\rho}_{\theta}^{\diamond} \bar{v}_{g}^{\diamond}$ of the geostrophic mass flux $\left.\bar{\rho}_{\theta} \bar{v}_{g}^{*}=\Pi\left(\bar{\rho}_{\theta}^{\diamond} \bar{v}_{g}^{\diamond}+{\overline{\check{\rho}_{\theta}}}_{\check{v}_{g}}\right)^{\delta}\right)$ can be identified with the two eddy flux terms on the righthand side of the balance condition (12).

Thus, in the geostrophic limit under convention II as under convention I, the Coriolis forces on the mass fluxes associated with the eddy fluxes of potential vorticity and of surface potential temperature balance eddy and mean components of the form drag. Conventions I and II differ in the way in which the form drag on isentropes is partitioned into mean and eddy components.

\section{d. Validity of balance condition in GCM simulation}

Figure 3 shows the mass flux streamfunction

$$
\Psi(\phi, \theta)=2 \pi a \cos (\phi) \int_{\theta_{b}}^{\theta} \bar{\rho}_{\theta} \bar{v}^{*} d \theta^{\prime}
$$

in the idealized GCM simulation. The mean meridional mass flux represented by the streamfunction is primarily composed of the mass flux associated with the eddy flux of potential vorticity (Fig. 4) and the mass flux associated with the surface pressure drag (Fig. 5). The vertical integral (11) of the mass flux associated with the surface pressure drag is what gives rise to the mass flux associated with the balanced eddy flux of surface potential temperature. 


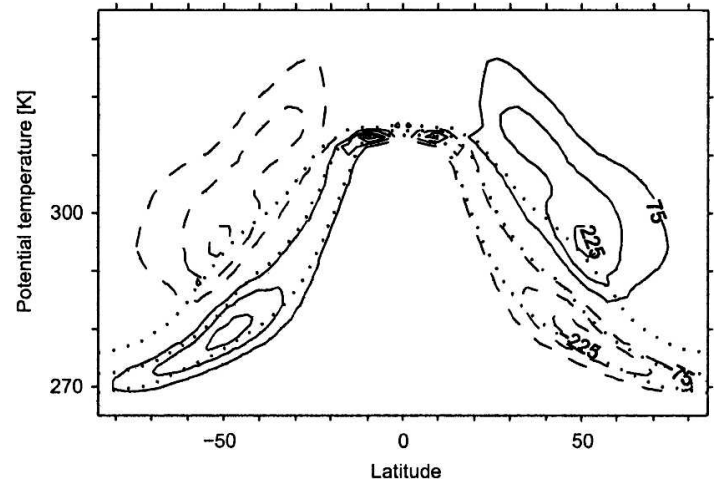

FIG. 4. Mass flux $-\bar{\rho}_{\theta} \overline{\hat{v}}^{*}{ }^{*} \bar{P}^{*}$ associated with eddy flux of potential vorticity under convention $\mathrm{I}\left(\mathrm{kg} \mathrm{m}^{-1} \mathrm{~K}^{-1} \mathrm{~s}^{-1}\right)$.

Under convention I, which we adopt for the remainder of this section, the mass flux associated with the eddy flux of potential vorticity changes sign near the top of the surface layer, from equatorward flux in the surface layer to poleward flux in the interior atmosphere (Fig. 4). The mass flux associated with the surface pressure drag is equatorward in the surface layer and vanishes in the interior atmosphere (Fig. 5). Taken together, these mass flux components result in poleward mass flux in the interior troposphere and equatorward mass flux near the surface, approximately within the surface layer (Fig. 3).

Figure 6 displays terms in the balance condition (15) with an approximate top of the surface layer as the upper limit $\theta_{i}(\phi)$ of the integration. The mass fluxes displayed thus are mass fluxes integrated over the surface layer. The approximate top of the surface layer is taken to be the $90 \%$ isoline of the cumulative distribution of surface potential temperatures (the uppermost dotted line in Figs. 3-5).

Figure 6a shows to what extent the balance condition (15) is quantitatively accurate at the approximate top of the surface layer. The integrated mass fluxes in Fig. 6a, representing the left-hand and right-hand side of the balance condition (15), differ by $10 \%-20 \%$. This differ-

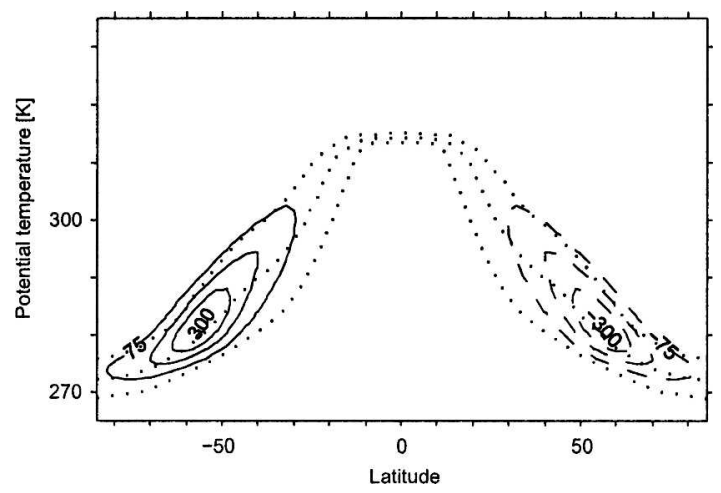

FIG. 5. Mass flux $\overline{\partial_{x} M \mathcal{H}} / \bar{P}^{*}=f \bar{v}_{g} / \bar{P}^{*} \approx \bar{\rho}_{\theta} \bar{v}_{g}$ associated with surface pressure drag under convention I $\left(\mathrm{kg} \mathrm{m}^{-1} \mathrm{~K}^{-1} \mathrm{~s}^{-1}\right)$. ence is due to four factors: (i) neglecting the diabatic component of the potential vorticity flux $(\$ 5 \%-10 \%$ error in midlatitudes; $10 \%-15 \%$ error in subtropics); (ii) taking the mass flux $-\bar{\rho}_{\theta}^{0} \overline{\tilde{v}_{s}^{\prime} \theta_{s}^{\prime}} s$ as representing, in place of the integral (11) of the mass flux associated with the surface pressure drag up to the top of the surface layer, the integral up to the approximate top of the surface layer ( $\$ 10 \%$ error); (iii) discretizing and interpolating to isentropic coordinates $(\$ 5 \%-10 \%$ error); (iv) approximating the integral (11) of the surface pressure drag term (in addition to the error due to the approximation of the upper limit of integration; $\$ 5 \%-$ $10 \%$ error).

Figure $6 \mathrm{~b}$ shows individual contributions to the mass flux integrated over the surface layer. The balanced eddy flux of surface potential temperature and the eddy flux of potential vorticity in the surface layer are associated with equatorward mass fluxes of similar magnitude, as is also evident from Figs. 4 and 5. [Since the mass flux associated with the eddy flux of potential vorticity changes sign below the $90 \%$ isoline of the cumulative distribution of surface potential temperatures (Fig. 4), some poleward mass flux contributes to the surface layer integral shown in Fig. 6b, thus reducing the magnitude of the equatorward mass flux shown there.] The Ekman mass flux is directed poleward in regions of surface westerlies and equatorward in regions of surface easterlies. In the extratropics, the Ekman mass flux is considerably weaker than the mass fluxes associated with the eddy fluxes of potential vorticity and surface potential temperature.

\section{e. Relative magnitude of mass fluxes associated with eddy fluxes}

Scaling estimates show that, under convention I, the mass fluxes associated with the eddy fluxes of surface potential temperature and potential vorticity in the surface layer are of the same order of magnitude not only in the simulation with the idealized GCM, but in general.

If the contribution of the relative vorticity to the potential vorticity is negligible (a good approximation in the surface layer), the mass flux associated with the eddy flux of potential vorticity is approximately equal to the eddy mass flux (16a). To obtain an estimate of the magnitude of this eddy mass flux integrated from the bottom $\theta_{b}$ to the top $\theta_{I}$ of the surface layer, we use the relation $\rho_{\theta}=-\left(\mathrm{g}^{-1} \partial_{\theta} p\right) \mathcal{H}\left(\theta-\theta_{s}\right)$ and ignore correlations between the pressure and the vertical shear of the meridional velocity on isentropes above the surface,

$$
\begin{aligned}
-\int_{\theta_{b}}^{\theta_{I}} \frac{\bar{\rho}_{\theta} \overline{\hat{v}}^{*}}{\bar{P}^{*}} d \theta & \approx \int_{\theta_{b}}^{\theta_{I}} \overline{\rho_{\theta}^{\prime} v^{\prime}} d \theta \\
& =-g^{-1} \int_{\theta_{b}}^{\theta_{I}} \overline{v^{\prime} \partial_{\theta} p \mathcal{H}} d \theta \\
& \sim-g^{-1} \frac{\tilde{v}_{s}^{\prime}\left[p\left(\theta_{I}\right)-p\left(\theta_{s}\right)\right] .}{}
\end{aligned}
$$



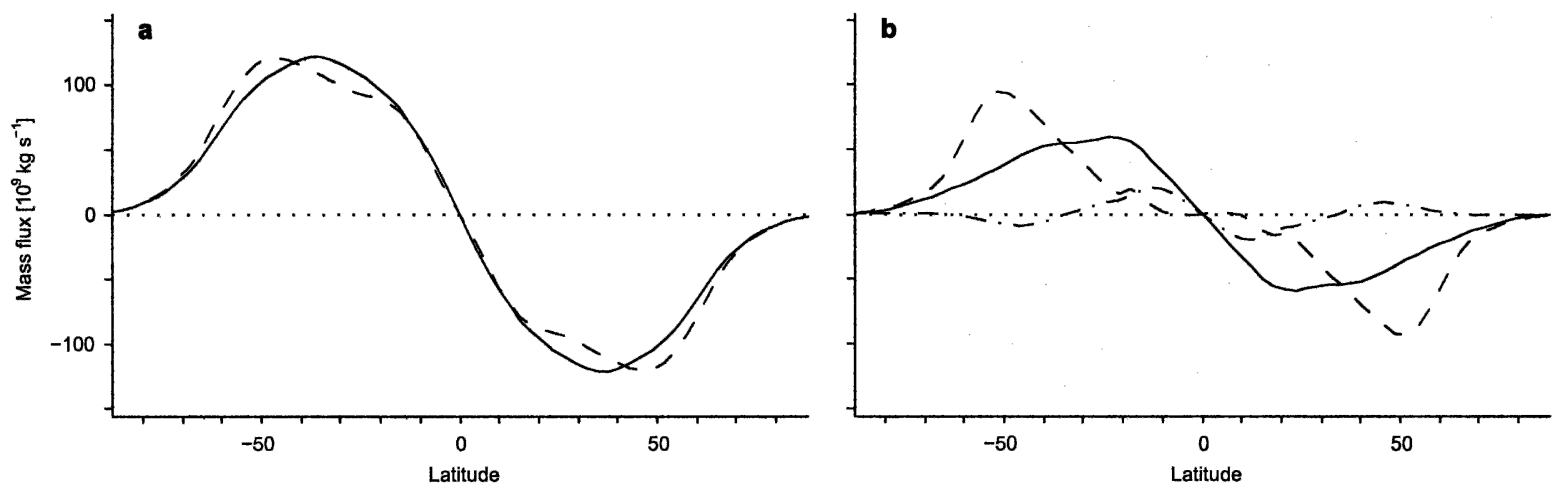

FIG. 6. Mean mass flux along isentropes integrated over surface layer (up to the $90 \%$ isoline of the cumulative distribution of surface potential temperatures). (a) Actual mass flux $\int \bar{\rho}_{\theta} \bar{v}^{*} d \theta$ (solid line) and mass flux associated with eddy fluxes and friction [dashed line; right-hand side of balance condition (15)]. (b) Individual contributions to mass flux under convention I: eddy flux of potential vorticity $-\int \bar{\rho}_{\theta} \hat{\hat{v} P}{ }^{*} / \bar{P}^{*} d \theta$ (solid line); balanced eddy flux of surface potential temperature $-\bar{\rho}_{\theta}^{0} \overline{\tilde{v}_{s}^{\prime} \theta_{s}^{\prime s}}$ (dashed line); Ekman mass flux $-\int \bar{\rho}_{\theta}{\overline{J_{F}^{y}}}_{F}^{*} / \bar{P}^{*} d \theta$ (dash-dotted line). The mass fluxes are multiplied by the lengths $2 \pi a \cos (\phi)$ of latitude circles, so that they are comparable with the values of the streamfunction in Fig. 3.

In the last line, we substituted the balanced eddy velocity $\tilde{v}_{s}^{\prime}$ at the surface as an estimate of a representative meridional eddy velocity on isentropes between the surface (potential temperature $\theta_{s}$ ) and the top of the surface layer (potential temperature $\theta_{I}$ ). Approximating the pressure at the top of the surface layer by expanding about the surface pressure and neglecting fluctuations of the isentropic density at the surface, one obtains for the instantaneous mass per unit area of the surface layer (cf. Juckes et al. 1994; Held and Schneider 1999)

$$
\left.g^{-1}\left[p\left(\theta_{I}\right)-p\left(\theta_{s}\right)\right] \sim g^{-1} \partial_{\theta} p\right|_{\theta_{s}}\left(\theta_{I}-\theta_{s}\right) \sim-\bar{\rho}_{\theta}^{s}\left(\theta_{I}-\theta_{s}\right),
$$

where $\bar{\rho}_{\theta}^{s}$ is the isentropic density averaged along the surface. Combining the estimates (20) and (21) and using the fact that the potential temperature $\theta_{I}$ at the top of the surface layer is fixed, one finds the estimate

$$
-\int_{\theta_{b}}^{\theta_{I}} \frac{\bar{\rho}_{\theta} \overline{\hat{v}}^{*}}{\bar{P}^{*}} d \theta \sim-\bar{\rho}_{\theta}^{s}{\overline{v_{s}^{\prime} \theta_{s}^{\prime}}}^{s}
$$

for the mass flux associated with the eddy flux of potential vorticity in the surface layer. The surface mean $\bar{\rho}_{\theta}^{s}$ of the isentropic density and the isentropic mean $\bar{\rho}_{\theta}^{0}$ at the mean surface potential temperature are of similar magnitude (the isentropic mean $\bar{\rho}_{\theta}^{0}$ is typically about a factor of 2 smaller than the surface mean $\bar{\rho}_{\theta}^{s}$ ). So the mass flux associated with the eddy flux of potential vorticity in the surface layer is of the same direction and order of magnitude as the mass flux associated with the balanced eddy flux of surface potential temperature.

By analyses of National Centers for Environmental Prediction-National Center for Atmospheric Research (NCEP-NCAR) reanalysis data (Kalnay et al. 1996) and analyses of idealized GCM simulations, including those described by Schneider (2004), it was verified that, in midlatitudes, the mass fluxes associated with the eddy fluxes of surface potential temperature and of potential vorticity in the surface layer indeed are generally of the same direction and order of magnitude. The analyzed simulations span a wide range of climates, from weakly baroclinic flows with pole-to-equator surface potential temperature differences of about $10 \mathrm{~K}$ to strongly baroclinic flows with pole-to-equator surface potential temperature differences of about $150 \mathrm{~K}^{4}$

\section{f. Qualitative account of mass flux along isentropes}

The mass flux streamfunction in isentropic coordinates is characterized by an overturning cell in each hemisphere, with equatorward mass flux in the surface layer and poleward mass flux aloft (Johnson 1989). With the simulation with the idealized GCM, we illustrate that qualitative aspects of the mass flux streamfunction in the extratropical troposphere can be understood by assuming that eddies tend to homogenize quantities that are materially conserved in adiabatic and inviscid flows (cf. Held and Schneider 1999).

In the interior of the extratropical troposphere, the mass flux along isentropes is primarily associated with the eddy flux of potential vorticity (Tung 1986). To the extent that eddies tend to homogenize potential vorticity on interior isentropes, the eddy flux $\hat{v}^{*}$ is

\footnotetext{
${ }^{4}$ As discussed in section $3 b$, the relative magnitude of the mass fluxes associated with the eddy fluxes of surface potential temperature and of potential vorticity in the surface layer depends on the conventions for the potential vorticity on surface-layer isentropes. Under convention II, it appears to be difficult to make general statements about the sign of the mass flux associated with the eddy flux of potential vorticity in the surface layer. Analyses of NCEP-NCAR reanalysis data and of simulations with the idealized GCM show that, under convention II, the mass flux associated with the eddy flux of potential vorticity in the surface layer can be equatorward or poleward.
} 
generally directed southward, since the mean potential vorticity gradient is generally positive in the interior troposphere (Fig. 1). According to the balance condition (15), a southward eddy flux of potential vorticity is associated with a poleward mass flux, consistent with the mass flux shown in Fig. 4.

In the surface layer of the extratropical atmosphere, the mass flux along isentropes is composed of the Ekman mass flux and the mass fluxes associated with the eddy fluxes of surface potential temperature and of potential vorticity in the surface layer.

(i) The mean surface potential temperature generally increases equatorward. To the extent that eddies tend to homogenize potential temperature along the surface, the eddy flux ${\overline{\tilde{v}_{s}^{\prime}}}^{\prime}{ }^{s}$ is therefore directed poleward. According to the balance condition (15), a poleward eddy flux of surface potential temperature is associated with an equatorward mass flux in the surface layer, consistent with the mass fluxes shown in Figs. 5 and $6 \mathrm{~b}$ (cf. Held and Schneider 1999).

(ii) The mean potential vorticity gradient under convention I is usually negative in the surface layer (Fig. 1). To the extent that eddies tend to homogenize potential vorticity in the surface layer, the eddy flux $\hat{v} \hat{P}^{*}$ is therefore directed northward. According to the balance condition (15), a northward eddy flux of potential vorticity implies an equatorward mass flux, consistent with the mass fluxes shown in Figs. 4 and 6b.

(iii) The Ekman mass flux is directed poleward in the region of the extratropical surface westerlies, hence is directed opposite to the net mass flux in the surface layer, and thus is weaker than the sum of the other components of the surface-layer mass flux.

Closer inspection of Figs. 3 and 4 reveals that the total mass flux and the mass flux associated with the eddy flux of potential vorticity in the surface layer are not distributed symmetrically about the mean surface potential temperature but are skewed to lower potential temperatures. This skewed distribution of mass fluxes appears to be due to the presence of a mixed layer near the surface, which implies a delta-function singularity of the isentropic density at the surface (Held and Schneider 1999). The distribution of mass fluxes in simulations with an idealized GCM that does not have a surface mixed layer is symmetric about the mean surface potential temperature (see Schneider 2004, Fig. 2).

\section{Comparison with quasigeostrophic theory}

\section{a. Continuously stratified models}

Rearranging the quasigeostrophic balance condition (9), one obtains the quasigeostrophic counterpart of the isentropic-coordinate balance condition (12),

$$
0=-\int_{0}^{\infty} \frac{\rho_{0} \overline{v_{g}^{\prime} q^{\prime}} z+\rho_{0} \overline{F^{x}}}{f_{0}} d z-\left.\frac{\rho_{0}}{\partial_{z} \theta_{0}} \overline{v_{g}^{\prime} \theta^{\prime}} z\right|_{z=0} .
$$

The eddy fluxes of quasigeostrophic potential vorticity along horizontal planes can be viewed as representing eddy fluxes of potential vorticity along isentropes (Charney and Stern 1962). Formally, the quasigeostrophic balance condition (22) thus resembles the isentropic-coordinate balance condition (12) if the integrals in the latter are likewise taken to extend to the top of the atmosphere. Near the surface, however, the two balance conditions generally differ.

In quasigeostrophic models of a continuously stratified atmosphere, potential vorticity gradients are usually positive throughout the atmosphere, down to immediately above the surface (see, e.g., Solomon and Stone 2001a, b). The eddy flux of quasigeostrophic potential vorticity in a statistically stationary state is directed downgradient in the mean, hence is negative, and is associated with a mass flux $-\rho_{0} \overline{v_{g}^{\prime} q^{\prime}} z / f_{0}$ that is directed poleward down to immediately above the surface. The equatorward mass flux $-\left.\left(\rho_{0} / \partial_{z} \theta_{0}\right) \overline{v_{g}^{\prime} \theta^{\prime}}\right|_{z=0}$ associated with the eddy flux of surface potential temperature closes the quasigeostrophic transformed Eulerian mean mass circulation in an infinitesimally thin sheet at the surface.

In contrast, the equatorward mass flux in isentropic coordinates is distributed over a surface layer that comprises up to one third of the mass of the Earth's troposphere. (According to NCEP-NCAR reanalysis data, the mean pressure at the $90 \%$ isoline of the cumulative distribution of surface potential temperatures varies between about 850 and $725 \mathrm{hPa}$ in the extratropics.) As we have seen, the Coriolis force on the mass flux associated with the eddy flux of potential vorticity in the surface layer balances, in the geostrophic limit, an eddy form drag. Irrespective of how eddy and mean fields are defined, this eddy form drag and the mass flux associated with the eddy flux of potential vorticity in the surface layer have no counterparts in quasigeostrophic models of a continuously stratified atmosphere because the horizontal planes to which the quasigeostrophic potential vorticity flux is confined do not intersect the surface.

The quasigeostrophic balance condition (22) can only be adequate if the mass of the surface layer is negligible. If vertical variations of density and static stability are negligible, the ratio $\eta$ of the mass of the surface layer to the mass of the troposphere scales as $\eta \sim$ $\left(\theta_{s}^{\prime} / \partial_{z} \bar{\theta}^{z}\right) / H_{t}$, where $\theta_{s}^{\prime}$ denotes a typical surface potential temperature fluctuation and $H_{t}$ the tropopause height. With surface potential temperature fluctuations that scale as $\theta_{s}^{\prime} \sim\left|\partial_{y} \bar{\theta}_{s}\right| L$, with a near-surface eddy length scale $L$, the condition that the mass of the surface layer is small is tantamount to the condition that the slope of isentropes near the surface is small, 


$$
\eta \sim\left|\frac{\partial_{y} \bar{\theta}_{s}}{\partial_{z} \bar{\theta}^{z}}\right| \frac{L}{H_{t}} \ll 1 .
$$

In quasigeostrophic scaling, the slope of isentropes and thus the ratio of the mass of the surface layer to the mass of the troposphere are assumed to be of order Rossby number. However in the earth's atmosphere, the ratio of the mass of the surface layer to the mass of the troposphere is generally greater than order Rossby number, so quasigeostrophic scaling is inaccurate.

Even when the mass of the surface layer is small, the way in which the isentropic-coordinate balance condition (12) represents potential vorticity dynamics and mass fluxes depends on the conventions for the potential vorticity on surface-layer isentropes. Under convention I, the mass flux associated with the eddy flux of potential vorticity is, in the geostrophic limit, equal to the geostrophic eddy mass flux $\overline{\rho_{\theta}^{\prime} v_{g}^{\prime}}$. The fluctuation $\rho_{\theta}^{\prime}$ of the isentropic density in the surface layer is generally not small: since the mean isentropic density $\bar{\rho}_{\theta}$ approaches zero at the bottom of the surface layer, in cold air outbreaks, during which isentropes near the bottom of the surface layer lie above the surface, the instantaneous isentropic density can be of the order of the mean isentropic density near the top of the surface layer, implying $\rho_{\theta}^{\prime} \gg \bar{\rho}_{\theta}$. As a consequence, the mass flux associated with the eddy flux of potential vorticity in the surface layer is not a third-order perturbation quantity - though one might expect it to be so given that it is integrated over the range of surface potential temperatures, a perturbation quantity in itself. The mass fluxes associated with the eddy fluxes of potential temperature and of potential vorticity in the surface layer are both second-order perturbation quantities (cf. section 3e). The mass flux associated with the eddy flux of potential vorticity in the surface layer, or the eddy form drag on surface-layer isentropes, is not negligible under convention I, even if the mass of the surface layer is small. Under convention II, in contrast, the mass flux associated with the eddy flux of potential vorticity is, in the geostrophic limit, equal to the geostrophic eddy mass flux $\check{\rho}_{\theta} \check{v}_{g} \diamond$. Under quasigeostrophic scaling, the fluctuations $\check{\rho}_{\theta}$ and $\breve{v}_{g}$ are first-order perturbation quantities, so the surface-layer integral of this mass flux is a thirdorder perturbation quantity and hence negligible. The potential vorticity dynamics and mass fluxes in continuously stratified quasigeostrophic models therefore resemble more closely the isentropic-coordinate potential vorticity dynamics and mass fluxes under convention II than under convention I.

\section{b. Two-layer model}

For the quasigeostrophic two-layer model, the counterpart of the isentropic-coordinate balance condition (12) is

$$
0=-\sum_{i=1}^{2} \frac{H_{i} \overline{v_{g i}^{\prime} q_{i}^{\prime}}+H_{i} \overline{F_{i}^{x}}}{f_{0}},
$$

where the index $i=1,2$ labels the lower and upper layer, $q_{i}=f_{0}+\beta_{0} y+\zeta_{g i}-f_{0} h_{i} / H_{i}$ is the quasigeostrophic potential vorticity, and $h_{i}$ is a layer-thickness fluctuation about a constant reference thickness $H_{i}$ [see, e.g., Held (2000) and Salmon (1998, chapter 2)]. The two layers can be viewed as representing the interior atmosphere and the surface layer in isentropic coordinates (Held 2000). The volume fluxes $-H_{i} \overline{\boldsymbol{v}_{g i}^{\prime} q_{i}^{\prime}} / f_{0}$ associated with the eddy fluxes of quasigeostrophic potential vorticity in the two layers correspond to the mass fluxes associated with the eddy fluxes of potential vorticity in the interior atmosphere and in the surface layer.

The quasigeostrophic potential vorticity dynamics and volume fluxes in the two-layer model resemble several aspects of the isentropic-coordinate potential vorticity dynamics and mass fluxes under convention I. The mean potential vorticity gradient (13) in isentropic coordinates is usually negative in the surface layer because the mean isentropic density $\bar{\rho}_{\theta}$ typically increases poleward along surface-layer isentropes; analogously, the mean quasigeostrophic potential vorticity gradient is negative in the lower layer of a typical baroclinically unstable two-layer model because the mean thickness perturbation $\bar{h}_{1}$ of the lower layer increases poleward (see, e.g., Held 2000). Both the mean isentropic density gradient in isentropic coordinates and the mean thickness gradient in the two-layer model reflect mean potential temperature gradients. In isentropic coordinates as in the two-layer model, the mean potential vorticity gradient typically changes sign between the surface layer and the interior atmosphere, or between the lower layer and upper layer, and downgradient mixing of potential vorticity implies that the potential vorticity fluxes and the associated mass or volume fluxes likewise change sign. However, the mass flux associated with the balanced eddy flux of surface potential temperature in the isentropic-coordinate balance condition (15) has no counterpart in the balance condition (23) for the two-layer model. From the perspective of the zonal momentum balance, in the geostrophic limit of the two-layer model, the Coriolis force on the volume flux associated with the eddy flux of quasigeostrophic potential vorticity balances the eddy form drag (Rhines and Holland 1979; Held 2000), analogous to the geostrophic limit in isentropic coordinates. But the mean form drag or mean surface pressure drag on surfacelayer isentropes has no counterpart in the two-layer model because the interface between the two layers never intersects the lower boundary (i.e., the layer thickness does not vanish in quasigeostrophic scaling).

The potential vorticity dynamics and volume fluxes in the quasigeostrophic two-layer model resemble the isentropic-coordinate potential vorticity dynamics and mass fluxes more closely under convention I than under 
convention II. For example, the mean potential vorticity gradient and the eddy flux of potential vorticity in isentropic coordinates under convention II do not necessarily change sign between the interior troposphere and surface layer, as they do under convention I and in the two-layer model.

\section{c. Shortcomings of quasigeostrophic theory}

Since the surface layer in the Earth's atmosphere comprises a significant fraction of the mass of the troposphere, the quasigeostrophic counterparts of the isentropic-coordinate balance condition (12) are not quantitatively accurate. However, quasigeostrophic theory may be qualitatively misleading in a way that goes beyond matters of quantitative accuracy.

Section $3 b$ has shown that, for closures of the eddy flux of potential vorticity in the surface layer, convention I is preferable to convention II. Under convention I, the isentropic-coordinate balance condition (15) contains three one-signed eddy flux terms: potential vorticity flux in the interior atmosphere, potential vorticity flux in the surface layer, and surface potential temperature flux. In contrast, its quasigeostrophic counterparts (22) and (23) each only contain two one-signed eddy flux terms. Continuously stratified quasigeostrophic models lack a representation of the potential vorticity flux in a surface layer of finite thickness. The quasigeostrophic two-layer model lacks a representation of the surface potential temperature flux.

The differences between the isentropic-coordinate balance condition (15) under convention I and its quasigeostrophic counterparts entail profound differences between the macroturbulent mean states that are realizable in the atmosphere and in quasigeostrophic models. The quasigeostrophic counterparts of the balance condition (15), with two one-signed eddy flux terms, imply strong constraints on the vertical structure of eddy diffusivities for potential vorticity and surface potential temperature. Substituting diffusive eddy flux closures $\overline{v_{g i}^{\prime} q_{i}^{\prime}}=-D_{i} \partial_{y} \bar{q}_{i}$ in the two-layer balance condition (23) and neglecting friction and relative vorticity contributions to the quasigeostrophic potential vorticity, one finds that, unless $\beta_{0}=0$, the eddy diffusivity $D_{1}$ in the lower layer must be greater than the eddy diffusivity $D_{2}$ in the upper layer (see, e.g., Vallis 1988; Treguier et al. 1997; Smith and Vallis 2002; Lapeyre and Held 2003). Similarly, substituting diffusive eddy flux closures $\overline{v_{g}^{\prime} q^{\prime}}=-D(z) \partial_{y} \bar{q}^{z}$ and $\left.\overline{v_{g}^{\prime} \theta^{\prime}}\right|_{z=0}=$ $-\left.D(0) \partial_{y} \bar{\theta}^{z}\right|_{z=0}$ in the continuously stratified balance condition (22), with an eddy diffusivity $D(z)$ for potential vorticity that is, at the surface $(z=0)$, equal to the eddy diffusivity for surface potential temperature, one finds that, unless $\beta_{0}=0$, the eddy diffusivity on average must decrease with height [I. Held (2002, personal communication); see Schneider (2004, footnote 6) for details]. Macroturbulent states of vertically uniform mixing, with eddy diffusivities that are constant in the vertical, are generally not realizable in quasigeostrophic models. In contrast, the isentropic-coordinate balance condition (15) under convention I, with three onesigned eddy flux terms, implies no such constraint on eddy diffusivities and is consistent with vertically uniform mixing. Not only are macroturbulent states of vertically uniform mixing realizable in the atmosphere, they also appear to be relevant for understanding the global circulation of the atmosphere (Schneider 2004).

The differences between the mean states that are realizable in the atmosphere and in quasigeostrophic models imply that, at least to the extent that convention I is appropriate for eddy flux closures, it may not be possible to carry over results on eddy flux closures from quasigeostrophic models to the atmosphere. This calls in question the relevance of quasigeostrophic models for the macroturbulence and general circulation of the atmosphere. As Charney put it in comments on his work on quasigeostrophic turbulence (Platzman 1990, p. 64):

The appearance of fronts vitiates these results [Charney's (1971) results on quasigeostrophic turbulence], and it is not known to what extent still. (...) One speaks of two-dimensional turbulence or quasigeostrophic turbulence, but I think that one always has to bear in mind that the results aren't in yet, because the real motions in real two-layer flows ... there's nothing to prevent the interface from intersecting the ground. It's only in quasigeostrophic flows that it doesn't. And I think when the interface intersects the ground, all bets are off, to a degree.

\section{Summary}

A form of the mean zonal momentum and potential vorticity balance was derived that holds throughout the flow domain in isentropic coordinates, including the surface layer of isentropes that sometimes intersect the surface. To derive mean balance equations, the instantaneous flow domain in isentropic coordinates was extended to isentropes with potential temperatures less than the surface potential temperature, following Lorenz (1955), by introducing massless isentropic layers inside the surface. The equations of motion on the massless isentropic layers reduced to the trivial statement $0=0$, which was formally dealt with by including Heaviside step functions in the equations. This formal device allowed averages, differentials, and integrals of terms in the mean zonal momentum balance, which can alternatively be viewed as a mean potential vorticity balance, to be written in a compact form using the rules of distributional calculus. Integrated vertically across isentropes, the mean zonal momentum or potential vorticity balance led to a balance equation involving the integrated potential vorticity flux, a balanced eddy flux of surface potential temperature, and frictional and diabatic processes. Within the approximations of the primitive equations, this balance equation holds for any 
ideal-gas atmosphere with stationary flow statistics. It generalizes similar balance equations well known in quasigeostrophic theory.

Decomposing the mean potential vorticity flux into a mean advective component and an eddy component yielded a balance condition that relates the mean mass flux along isentropes to eddy fluxes of potential vorticity and of surface potential temperature. For the extratropical interior troposphere, the balance condition implies that, as is well known, the southward eddy flux of potential vorticity is associated with a poleward mass flux. For the surface layer, the balance condition depends on conventions for the potential vorticity on isentropes inside the surface. Under convention I, the balance condition implies for the extratropical surface layer that both a northward eddy flux of potential vorticity and the poleward eddy flux of surface potential temperature contribute to an equatorward mass flux. Under convention II, the balance condition still implies that an eddy flux of potential vorticity contributes to the mass flux in the extratropical surface layer, but it appears to be difficult to make general statements about the sign of the eddy flux of potential vorticity in the surface layer. Focusing on convention I, which appears to be convenient for eddy flux closures, we showed by means of simulations with an idealized GCM that the balance condition is quantitatively accurate. The simulations confirmed scaling estimates suggesting that, under convention I, the eddy fluxes of surface potential temperature and of potential vorticity in the surface layer are associated with mass fluxes of similar magnitude. In the geostrophic limit, the Coriolis forces on these mass fluxes were shown to balance eddy and mean components of the form drag on surfacelayer isentropes, the mean component of the form drag representing a mean surface pressure drag due to the intersection of isentropes with the surface. Conventions I and II differ in the way in which the form drag on isentropes is partitioned into mean and eddy components.

Quasigeostrophic models take into account only one of the two eddy flux terms that contribute to the mass flux in the isentropic-coordinate surface layer. The potential vorticity flux in the surface layer, or the eddy form drag on surface-layer isentropes, appears in the lower layer of the two-layer model, which does not take into account that surface-layer isentropes sometimes intersect the surface (i.e., the thickness of the lower layer sometimes vanishes). The surface potential temperature flux, or the mean form drag, appears in continuously stratified models, which do not take into account that the surfaces to which the potential vorticity flux is confined sometimes intersect the surface (i.e., the surface layer has finite thickness). Both terms do not appear simultaneously in quasigeostrophic models. Under convention II, if the Rossby number and the mass of the surface layer are small-the latter a condition tantamount to the quasigeostrophic condition that the slope of isentropes is small-the eddy flux of potential vorticity in the surface layer is negligible, and the quasigeostrophic balance condition represents the potential vorticity dynamics and mass fluxes in isentropic coordinates adequately. In the earth's atmosphere, however, the surface layer comprises a significant fraction of the mass of the troposphere, so quasigeostrophic scaling is inaccurate. Under convention I, moreover, eddy fluxes satisfy different balance conditions in quasigeostrophic models than in the atmosphere, even when the mass of the surface layer is small. To the extent that convention I is appropriate for eddy flux closures, this calls into question the relevance of quasigeostrophic theories for the macroturbulence and global circulation of the atmosphere. Quasigeostrophic theory may be overconstrained in that it does not adequately take the intersection of isentropes with the surface into account.

Acknowledgments. I am grateful to Chris Walker for performing the simulations with the idealized GCM and for providing analyses of observational data with which I verified the scaling estimates of section $3 e$; to Isaac Held for discussions that helped to clarify commonalities and differences between dynamics in isentropic coordinates and in quasigeostrophic theory; to Tieh-Yong Koh and Peter Haynes for comments on drafts of this paper; and to the Davidow Research Fund for financial support. The balance condition (12) was originally included in a section of a paper jointly authored with Isaac Held and Stephen Garner (Schneider et al. 2003), a section that was later removed to reduce the length of that paper. I thank Isaac Held and Stephen Garner for helpful discussions during the writing of that section.

\section{APPENDIX A}

\section{Idealized GCM and Computation of Flow Statistics}

The idealized GCM is a primitive equation model of an ideal-gas atmosphere with a spherical lower boundary. Radiative processes are represented in an idealized fashion as Newtonian relaxation of temperatures toward an axially and hemispherically symmetric radiative equilibrium state. A simple convection scheme that relaxes temperatures toward a temperature profile with fixed lapse rate $0.7 \Gamma_{d}$, where $\Gamma_{d}=-g / c_{p}$ is the dry adiabatic lapse rate, mimics dynamic heating due to moist convection. As a parameterization of subgridscale turbulent mixing, vertical diffusion of momentum, and temperature is included in a planetary boundary layer of fixed height $2.5 \mathrm{~km}$ (Smagorinski et al. 1965). The primitive equations are integrated with the spectral transform method with T42 horizontal resolution and 30 vertical $\sigma$ levels. See Schneider (2004) for a description of a model that differs from the present model only in the parameterization of moist convection and boundary layer processes. 
Isentropic flow statistics were obtained by sampling flow quantities 4 times per simulated day and interpolating them from the $\sigma$ coordinate levels of the GCM to 100 isentropic coordinate levels. The isentropic levels are equally spaced in the transformed potential temperature coordinate $\theta^{-1 / \kappa}$, with the lowermost and uppermost isentropic levels corresponding to potential temperatures of 244 and $408 \mathrm{~K}$. For the computation of isentropic mass fluxes, the instantaneous meridional mass flux in $\sigma$ coordinates was interpolated columnwise and linearly in $\theta^{-1 / \kappa}$ to isentropic coordinates, in such a way that vertical integrals of the instantaneous meridional mass flux in $\sigma$ coordinates were equal to the corresponding vertical integrals of the interpolated meridional mass flux in isentropic coordinates (Juckes et al. 1994). For the computation of other flow statistics, flow quantities were interpolated from $\sigma$ coordinates to isentropic coordinates using a locally monotonic Hermite interpolant that is piecewise cubic in $\theta^{-1 / \kappa}$ (Fritsch and Butland 1984).

\section{APPENDIX B}

\section{Notation and Symbols}

$\partial_{\xi} \quad$ Partial derivative with respect to coordinate $\xi$ (with horizontal derivatives understood as derivatives along isentropes if argument depends on a vertical coordinate)

$\overline{(\cdot)} \quad$ Temporal and zonal mean (along isentropes if argument depends on a vertical coordinate)

$\overline{(\cdot)}^{*} \quad$ Isentropic mean $\overline{\left(\rho_{\theta} \cdot\right)} / \bar{\rho}_{\theta}$ weighted by isentropic density $\rho_{\theta}$

(.) Fluctuation about density-weighted isentropic mean $\overline{(\cdot)} *$

$\overline{(\cdot)} \quad$ Isentropic mean $\overline{(\cdot \mathscr{H})} / \Pi$ weighted by step function $\mathcal{H}$, with $\overline{\mathcal{H}}=\Pi$

$($.$) Fluctuation about step-function-weighted isen-$ tropic mean $\overline{(\cdot)} \diamond$

$\overline{(\cdot)}^{s} \quad$ Temporal and zonal mean along surface

$\overline{(\cdot)}^{z} \quad$ Temporal and zonal mean along horizontal planes

$(\cdot)^{\prime} \quad$ Fluctuation about surface mean $\overline{(\cdot)}^{s}$, about isentropic mean $\overline{(\cdot)}$, or about horizontal mean $\overline{(\cdot)}^{z}$

a Planet radius

$B \quad$ Bernoulli function $B=1 / 2\left(u^{2}+v^{2}\right)+c_{p} T+g z$

$c_{p} \quad$ Specific heat at constant pressure

$f, f_{0} \quad$ Coriolis parameter $f=2 \Omega \sin (\phi)$, constant reference value

$F^{x} \quad$ Zonal component of frictional force per unit mass

g Gravitational acceleration

$H_{t} \quad$ Tropopause height

$\mathcal{H}(\cdot) \quad$ Heaviside step function, with shorthand $\mathcal{H}=$ $\mathcal{H}\left(\theta-\theta_{s}\right)$

$J_{Q}^{y}, J_{F}^{y}$ Diabatic and frictional components (5) of meridional potential vorticity flux

$L \quad$ Length scale
$M \quad$ Montgomery streamfunction/dry static energy $M=c_{p} T+g z$

$p, p_{r} \quad$ Pressure, constant reference pressure

PVU Potential vorticity unit $1 \mathrm{PVU}=10^{-6} \mathrm{~K} \mathrm{~m}^{2} \mathrm{~kg}^{-1} \mathrm{~s}^{-1}$

$P \quad$ Potential vorticity

$P_{g} \quad$ Potential vorticity $P_{g}=f / \rho_{\theta}$ in geostrophic limit

$q \quad$ Quasigeostrophic potential vorticity

$Q$ Material derivative of potential temperature $Q=$ $D \theta / D t$

$R \quad$ Gas constant

Ro $\quad$ Rossby number Ro $=U /(f L)$

$t \quad$ Time

$T, T_{r}$ Temperature, constant reference temperature

$u, v$ Horizontal velocity components (eastward, northward)

$U \quad$ Horizontal velocity scale

$\tilde{v}_{s}^{\prime} \quad$ Balanced meridional eddy velocity (7) at surface

$v_{g} \quad$ Geostrophic meridional velocity

$x, y, z$ Local Cartesian coordinates (eastward, northward, upward)

$\beta, \beta_{0}$ Meridional derivative $\beta=2 \Omega a^{-1} \cos (\phi)$ of Coriolis parameter, constant reference value

$\delta(\cdot) \quad$ Dirac delta function

$\zeta_{\theta} \quad$ Relative vorticity component normal to isentropes

$\zeta_{g} \quad$ Relative vorticity of geostrophic flow

$\theta, \theta_{r}$ Potential temperature, constant reference potential temperature

$\theta_{0} \quad$ Reference potential temperature profile $\theta_{0}(z)$

$\theta_{b} \quad$ Potential temperature less than or equal to the lowest potential temperature that occurs at surface

$\theta_{i} \quad$ Potential temperature greater than or equal to the highest potential temperature that occurs at a given latitude at surface

$\theta_{I} \quad$ Potential temperature at top of surface layer

$\kappa \quad$ Adiabatic exponent $\kappa=R / c_{p}$

$\Pi \quad$ Cumulative distribution function $\Pi(y, \theta)$ of surface potential temperatures

$\rho, \rho_{r} \quad$ Density, constant reference density

$\rho_{0} \quad$ Reference density profile $\rho_{0}(z)$

$\rho_{\theta} \quad$ Isentropic density $\rho_{\theta}=-\left(g^{-1} \partial_{\theta} p\right) \mathcal{H}\left(\theta-\theta_{s}\right)$

$\bar{\rho}_{\theta}^{0} \quad$ Mean isentropic density $\bar{\rho}_{\theta}^{0}=\bar{\rho}_{\theta}\left(\bar{\theta}_{s}\right)$ at mean surface potential temperature $\bar{\theta}_{s}(y)$

$\sigma \quad$ Terrain-following coordinate $\sigma=p / p_{s}$

$\phi \quad$ Latitude

$\Omega \quad$ Angular velocity of planetary rotation

\section{REFERENCES}

Andrews, D. G., 1983: A finite-amplitude Eliassen-Palm theorem in isentropic coordinates. J. Atmos. Sci., 40, 1877-1883.

_ , and M. E. McIntyre, 1976: Planetary waves in horizontal and vertical shear: The generalized Eliassen-Palm relation and the mean zonal acceleration. J. Atmos. Sci., 33, 2031-2048.

, J. R. Holton, and C. B. Leovy, 1987: Middle Atmosphere Dynamics. International Geophysics Series, Vol. 40, Academic Press, 489 pp. 
Charney, J. G., 1971: Geostrophic turbulence. J. Atmos. Sci., 28, 1087-1095.

— jets in a rotating atmosphere. J. Atmos. Sci., 19, 159-172.

Corrsin, S., 1974: Limitations of gradient transport models in random walks and in turbulence. Advances in Geophysics, Vol. 18A, Academic Press, 25-60.

Edmon, H. J., B. J. Hoskins, and M. E. McIntyre, 1980: EliassenPalm cross sections for the troposphere. J. Atmos. Sci., 37, 2600-2616.

Fritsch, F. N., and J. Butland, 1984: A method for constructing local monotone piecewise cubic interpolants. SIAM J. Sci. Stat. Comput., 5, 300-304.

Gallimore, R. G., and D. R. Johnson, 1981: The forcing of the meridional circulation of the isentropic zonally averaged circumpolar vortex. J. Atmos. Sci., 38, 583-599.

Green, J. S. A., 1970: Transfer properties of the large-scale eddies and the general circulation of the atmosphere. Quart. J. Roy. Meteor. Soc., 96, 157-185.

Haynes, P. H., and M. E. McIntyre, 1987: On the evolution of vorticity and potential vorticity in the presence of diabatic heating and frictional or other forces. J. Atmos. Sci., 44, 828841 .

- and - 1990: On the conservation and impermeability theorems for potential vorticity. J. Atmos. Sci., 47, 2021-2031.

Held, I. M., 2000: The general circulation of the atmosphere. Proc. Summer Program in Geophysical Fluid Dynamics, Woods Hole, MA, Woods Hole Oceanographic Institution. [Available online at http://gfd.whoi.edu/proceedings/2000/ PDFvol2000.html.]

_- and B. J. Hoskins, 1985: Large-scale eddies and the general circulation of the troposphere. Advances in Geophysics, Vol. 28A, Academic Press, 3-31.

— , and T. Schneider, 1999: The surface branch of the zonally averaged mass transport circulation in the troposphere. $J$. Atmos. Sci., 56, 1688-1697.

Hoskins, B. J., 1991: Towards a PV- $\theta$ view of the general circulation. Tellus, 43 AB, 27-35.

Johnson, D. R., 1980: A generalized transport equation for use with meteorological coordinate systems. Mon. Wea. Rev., 108, 733-745

, 1989: The forcing and maintenance of global monsoonal circulations: An isentropic analysis. Advances in Geophysics, Vol. 31, Academic Press, 43-304.

Juckes, M. N., I. N. James, and M. Blackburn, 1994: The influence of Antarctica on the momentum budget of the southern extratropics. Quart. J. Roy. Meteor. Soc., 120, 1017-1044.

Kalnay, E., and Coauthors, 1996: The NCEP/NCAR 40-Year Reanalysis Project. Bull. Amer. Meteor. Soc., 77, 437-471.
Koh, T.-Y., and R. A. Plumb, 2004: Isentropic zonal average formulation and the near-surface circulation. Quart. J. Roy. Meteor. Soc., 130, 1631-1654.

Lapeyre, G., and I. M. Held, 2003: Diffusivity, kinetic energy dissipation, and closure theories for the poleward eddy heat flux. J. Atmos. Sci., 60, 2907-2916.

Lorenz, E. N., 1955: Available potential energy and the maintenance of the general circulation. Tellus, 7, 157-167.

Peixoto, J. P., and A. H. Oort, 1992: Physics of Climate. American Institute of Physics, $520 \mathrm{pp}$.

Platzman, G. W., 1990: The atmosphere-A challenge. The Atmosphere-A Challenge. The Science of Jule Gregory Charney, R. S. Lindzen, E. N. Lorenz, and G. W. Platzman, Eds. Historical Monograph Series, Amer. Meteor. Soc., 11-82.

Rhines, P. B., and W. R. Holland, 1979: A theoretical discussion of eddy-driven mean flows. Dyn. Atmos. Oceans, 3, 289-325.

Salmon, R., 1998: Lectures on Geophysical Fluid Dynamics. Oxford University Press, 378 pp.

Schneider, T., 2004: The tropopause and the thermal stratification in the extratropics of a dry atmosphere. J. Atmos. Sci., 61, 1317-1340.

—, I. M. Held, and S. T. Garner, 2003: Boundary effects in potential vorticity dynamics. J. Atmos. Sci., 60, 1024-1040.

Smagorinski, J., S. Manabe, and J. J. L. Holloway, 1965: Numerical results from a nine-level general circulation model of the atmosphere. Mon. Wea. Rev., 93, 727-768.

Smith, K. S., and G. K. Vallis, 2002: The scales and equilibration of midocean eddies: Forced-dissipative flow. J. Phys. Oceanogr., 32, 1699-1720.

Solomon, A., and P. H. Stone, 2001a: Equilibration in an eddy resolving model with simplified physics. J. Atmos. Sci., 58, $561-574$.

- and $-2001 \mathrm{~b}$ : A study of the sensitivity of the equilibrated state of an intermediate model of the midlatitude troposphere to changes in radiative forcing. J. Atmos. Sci., 58 , 2395-2410.

Treguier, A. M., I. M. Held, and V. D. Larichev, 1997: On the parameterization of quasigeostrophic eddies in primitive equation ocean models. J. Phys. Oceanogr., 27, 567-580.

Tung, K. K., 1986: Nongeostrophic theory of zonally averaged circulation. Part I: Formulation. J. Atmos. Sci., 43, 2600-2618.

Vallis, G. K., 1988: Numerical studies of eddy transport properties in eddy-resolving and parameterized models. Quart. J. Roy. Meteor. Soc., 114, 183-204.

Yang, H., K. K. Tung, and E. Olaguer, 1990: Nongeostrophic theory of zonally averaged circulation. Part II: Eliassen-Palm flux divergence and isentropic mixing coefficient. J. Atmos. Sci., 47, 215-241. 